Check for updates

Cite this: RSC Adv., 2018, 8, 29321

\title{
Photo-responsive polymeric micelles and prodrugs: synthesis and characterization
}

\author{
Shiu-Wei Wang, ${ }^{a}$ Yin-Ku Lin, ${ }^{b}$ Jia-You Fang ${ }^{c}$ and Ren-Shen Lee (D) *a
}

Bio-recognizable and photocleavable amphiphilic glycopolymers and prodrugs containing photodegradable linkers (i.e. 5-hydroxy-2-nitrobenzyl alcohol) as junction points between biorecognizable hydrophilic glucose (or maltose) and hydrophobic poly( $\alpha$-azo- $\varepsilon$-caprolactone)-grafted alkyne or drug chains were synthesized by combining ring-opening polymerization, nucleophilic substitution, and "click" post-functionalization with alkynyl-pyrene and 2-nitrobenzyl-functionalized indomethacin (IMC). The block-grafted glycocopolymers could self-assemble into spherical photoresponsive micelles with hydrodynamic sizes of $<200 \mathrm{~nm}$. Fluorescence emission measurements indicated the release of Nile red, a hydrophobic dye, encapsulated by the Glyco-ONB-P $\left(\alpha N_{3} C L-g\right.$ alkyne $)_{n}$ micelles, in response to irradiation caused by micelle disruption. Light-triggered bursts were observed for IMC-loaded or -conjugated micelles during the first $5 \mathrm{~h}$. Following light irradiation, the drug release rate of IMC-conjugated micelles was faster than that of IMC-loaded micelles. Selective lectin binding experiments confirmed that glycosylated Glyco-ONB-P( $\alpha N_{3} \mathrm{CL}-g$-alkyne $)_{n}$ could be used in biorecognition applications. The nano-prodrug with and without UV irradiation was associated with negligible levels of toxicity at concentrations of less than $30 \mu \mathrm{g} \mathrm{mL}^{-1}$. The confocal microscopy and flow cytometry results indicated that the uptake of doxorubicin (DOX)-loaded micelles with UV irradiation by HeLa cells was faster than without UV irradiation. The DOX-loaded Gluco-ONB-P( $\alpha N_{3} C L-g$-PONBIMC $)_{10}$ micelles effectively inhibited HeLa cells' proliferation with a half-maximal inhibitory concentration of 8.8 $\mu \mathrm{g} \mathrm{mL}{ }^{-1}$.

rsc.li/rsc-advances

\section{Introduction}

In recent years, multifarious drug delivery systems (e.g., polymeric micelles, polymer drug conjugates, and polymeric nanoparticles) have been developed to address the problems associated with drug molecules, such as low aqueous solubility, short plasma circulation time, rapid in vivo degradation and systemic toxicity. ${ }^{1}$ Compared with free drugs, drugs incorporated into polymeric micelles exhibit favorable therapeutic advantages: enhanced drug solubility in water, prolonged circulation time by inhibiting phagocytic and renal clearance, and passive targeting to the tumor tissues by the enhanced permeability and retention effect. ${ }^{2-5}$ However, many drug delivery systems face strong limitations that potentially affect their further translation to clinical tests: the burst release in which a large fraction of adsorbed drug is rapidly released after administration and the inadequate drug loading which usually necessitates a high concentration of nanocarriers to obtain

${ }^{a}$ Division of Natural Science, Center of General Education, Chang Gung University, 259 Wen-Hwa 1st Road, Guishan Dist., Tao-Yuan 33302, Taiwan. E-mail: shen21@mail. cgu.edu.tw

${ }^{b}$ Department of Traditional Chinese Medicine, Chang Gung Memorial Hospital at Keelung, Keelung, Taiwan

${ }^{c}$ Graduate Institute of Natural Products, Chang Gung University, Tao-Yuan, Taiwan a noticeable therapeutic effect. These major obstacles may be overcome by applying the polymer prodrug approach, where the drug is covalently conjugated onto polymer backbones and side chains through labile linkers that can be cleaved in certain conditions. The drugs cannot be released before the degradable linking is cleaved. Therefore, polymer drug conjugates exhibit excellent storage stability, low systemic toxicity in circulation, and localized drug releases. ${ }^{6,7}$

Stimulus-responsive micelles formed from amphiphilic copolymers or polymer drug conjugates are promising materials for drug delivery and may lead to enhanced biological properties and efficiencies. ${ }^{8-15}$ Among the available stimuli, light is of particular interest because it can be highly localized in time and space. ${ }^{16}$ Photoactivable drug delivery systems are not only suitable for surface cancer treatment but also applicable to deep-seated cancer under endoscopic or optical fiber guidance, which is also used for photodynamic therapy. ${ }^{17-20}$ Recently, several research groups have demonstrated an irreversible response, involving the incorporation of the photocleavable units in the main chain of one of the blocks or in the hydrophobic cores conjugated with photocaged chemotherapeutic drugs, that induces the selective degradation of a specific micellar compartment. Among the many studied photolabile groups, o-nitrobenzyl (ONB) alcohol derivatives have received 
considerable attention in the area of synthetic polymer and polymer drug conjugates. ${ }^{21-23}$

In this study, we present a synthetic platform, which permits in situ construction of various light-triggered drug delivery systems from amphiphilic glycose block functionalized poly $\left(\alpha\right.$-azo- $\varepsilon$-caprolactone) (Glyco-ONB-P $\left.\alpha \mathrm{N}_{3} \mathrm{CL}_{n}\right)$ copolymers with photocleavable ortho-nitrobenzyl (ONB) units in the middle moiety and side chain, in which the drug molecules to be delivered can be loaded either physically (i.e., the encapsulation approach) or chemically attached (i.e., the prodrug approach). For the encapsulation approach, the ONB linked the hydrophilic block glycose and the hydrophobic block $\mathrm{P}\left(\alpha \mathrm{N}_{3} \mathrm{CL}\right)_{n}$ with the grafted alkyne, whereas the prodrugs were obtained by incorporating the linkers between the Glyco-ONB$\mathrm{P} \mathrm{N}_{3} \mathrm{CL}_{n}$ and conjugating a drug molecule in the hydrophobic block. A potent nonsteroidal anti-inflammatory drug, indomethacin (IMC), is suitable drug model for studying micelle formation. ${ }^{24}$ The block-graft copolymers were synthesized through ring-opening polymerization (ROP), nucleophilic substitution, and "click" reactions (Scheme 1). The physicochemical and photodegradable properties of these micelles in the aqueous phase were examined through fluorescence spectroscopy, dynamic light scattering (DLS), and transmission electron microscopy (TEM). The phototriggered controlled release from the drug-loaded micelles and micelle drug conjugates in the physiological condition was reported. Finally, to demonstrate their potential as fluorescent probes in optical bioimaging, these DOX-encapsulated prodrug micelles were internalized in the human cervical cancer cell line HeLa and analyzed through fluorescence imaging and cytotoxicity study.

\section{Experimental section}

\section{Materials}

2-Chlorocyclohexanone (99\%), 5-hydroxy-2-nitrobenzyl alcohol (97\%), 1-hexyne, 1-decayne, sodium azide, pyrene (99\%), dimethylamino-pyridine (DMAP, 99\%), $N, N^{\prime}$-dicyclohexylcarbodiimide (DCC, >99\%), IMC (99\%), and Nile red (NR, 98\%) were purchased from Aldrich Chemical Co. (Milwaukee, WI, USA). Moreover, $m$-chloroperoxybenzoic acid, $\alpha$-Dpentaacetyl-glucopyranoside, $\mathrm{D}-(+)$-maltose, and boron trifluoride diethyl etherate were purchased from Fluka Chemical Co. (Buchs, Switzerland). Stannous octoate $\left(\right.$ SnOct $\left._{2}, 95 \%\right)$ was purchased from Strem Chemical Inc. (Newburyport, MA, USA). 2-Chloro- $\varepsilon$-caprolactone ( $\alpha$-ClCL), 3-bromo-propyl-glucose, 3bromo-propyl-maltose, 2-propargyoxymethyl were prepared according to previously described methods, ${ }^{22,25}$ but with modification. Doxorubicin hydrochloride (99\%; Aldrich, Saint Louis, MO, USA) was deprotonated to obtain hydrophobic DOX as described previously. ${ }^{26} \mathrm{~N}, \mathrm{~N}$-Dimethyl formamide (DMF) and toluene were distilled under calcium hydride. Other highpressure liquid chromatography (HPLC) grade solvents, such as tetrahydrofuran (THF), dimethylsulfoxide (DMSO), methanol, chloroform $\left(\mathrm{CHCl}_{3}\right)$, ethyl acetate (EA), and $n$-hexane were purchased from Merck KGaA (Darmstadt, Germany). A Milli-Q Plus system (Waters, Milford, MA, USA) was used to obtain ultrapure water. Dulbecco's modified Eagle's medium (DMEM), trypsin/EDTA, $100 \times$ antibiotic antimycotic, and Hoechst 33342 nuclei dye were purchased from Gibco (Invitrogen Corp. Carlsbad, CA, USA). Fetal bovine serum (FBS) was obtained from Biological Industry (Kibbutz Beit Haemek, Israel). A CellTiter 96® AQueuous One Solution kit was obtained from Promega

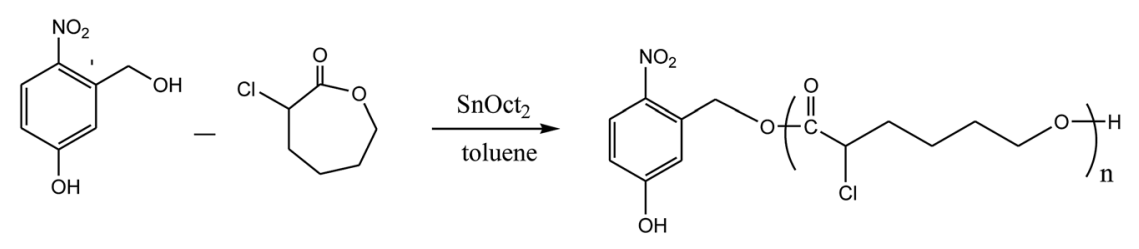

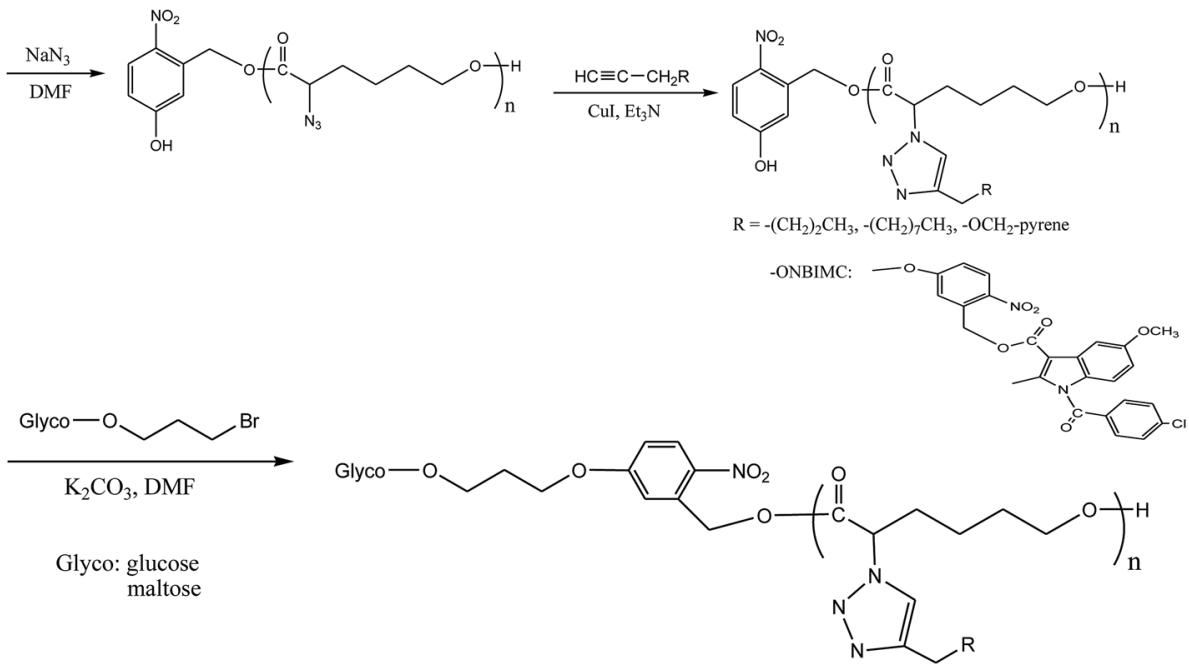

Scheme 1 Synthesis photo-triggered Glyco-ONB-P( $\alpha \mathrm{N}_{3} \mathrm{CL}-\mathrm{g}$-alkyne $)_{n}$ and prodrug. 
(A)

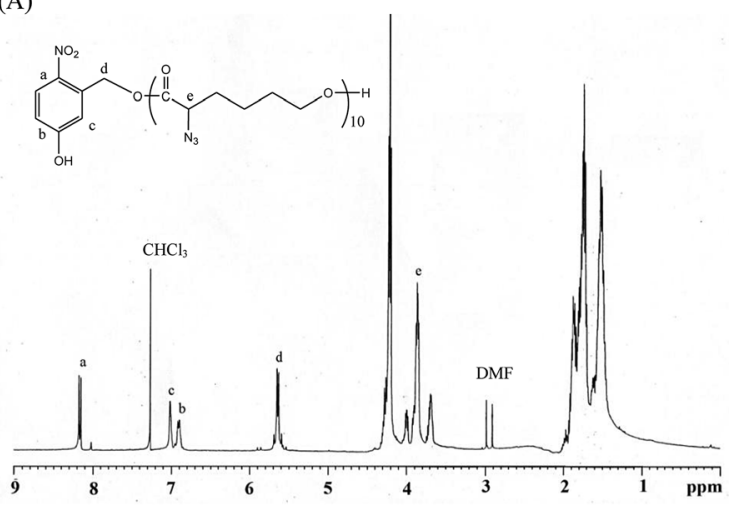

(C)

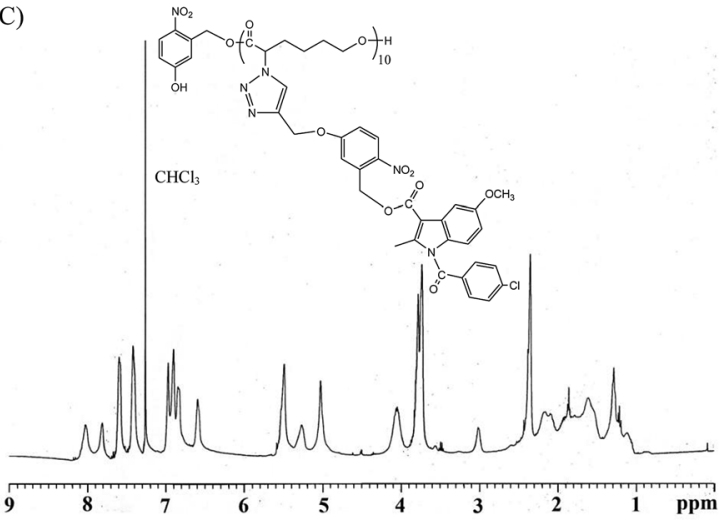

(B)
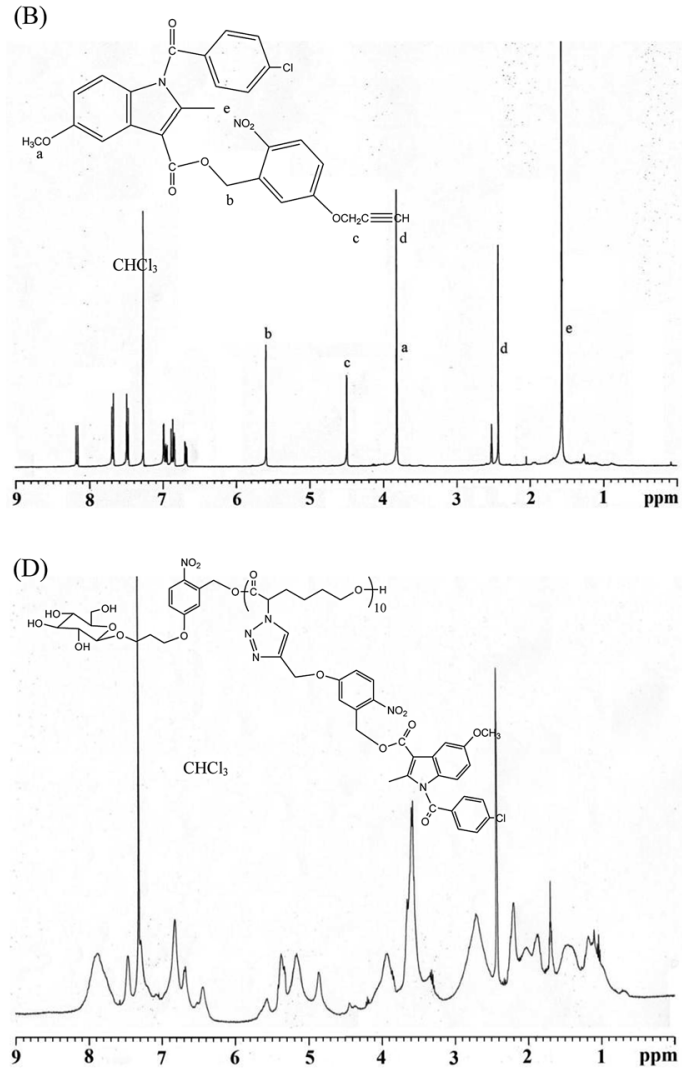

Fig. 1 Representative ${ }^{1} \mathrm{H}$ NMR spectroscopy: (A) HONB-P( $\left.\alpha N_{3} C L\right)_{10}$, (B) (4-propargoxy-2-nitro)benzyl indomethacinoate (PONBIMC), (C) $\mathrm{HONB}-\mathrm{P}\left(\alpha \mathrm{N}_{3} \mathrm{CL}-\mathrm{g}-\mathrm{PONBIMC}\right)_{10}$, and (D) Gluco-ONB-P( $\left.\alpha \mathrm{N}_{3} \mathrm{CL}-g-\mathrm{PONBIMC}\right)_{10}$.

(Fitchburg, WI, USA) Amiloride, chlorpromazine, methyl- $\beta$ cyclodextrin, and nystatin were purchased from Sigma Aldrich (Saint Louis, MO, USA).

Prepare of 2-propargyoxymethyl pyrene (Ppyrene). Pyrenemethanol ( $2 \mathrm{~g}, 8.61 \mathrm{mmol})$ was dissolved in toluene $(40 \mathrm{~mL})$ and added the $\mathrm{KOH}(9.64 \mathrm{~g}, 171.8 \mathrm{mmol})$. To the resulting suspension was slowly added propargyl bromide (25.6 g, 172.20 $\mathrm{mmol}$ ) and stirred at $60{ }^{\circ} \mathrm{C}$ for $60 \mathrm{~h}$. Then, the mixture was filtered and concentrated in vacuo. The residue was purified by flash column chromatography with hexane/EA $(5: 1)$ to give 2 propargyoxymethyl pyrene $\left(1.51 \mathrm{~g}, 65 \%\right.$ yield). ${ }^{1} \mathrm{H}$ NMR $\left(\mathrm{CDCl}_{3}\right.$, $\delta): 8.01-8.49(\mathrm{~m}, 9 \mathrm{H}), 5.35(\mathrm{~s}, 2 \mathrm{H}), 4.29(\mathrm{~d}, 2 \mathrm{H}), 2.48(\mathrm{~d}, 1 \mathrm{H})$.

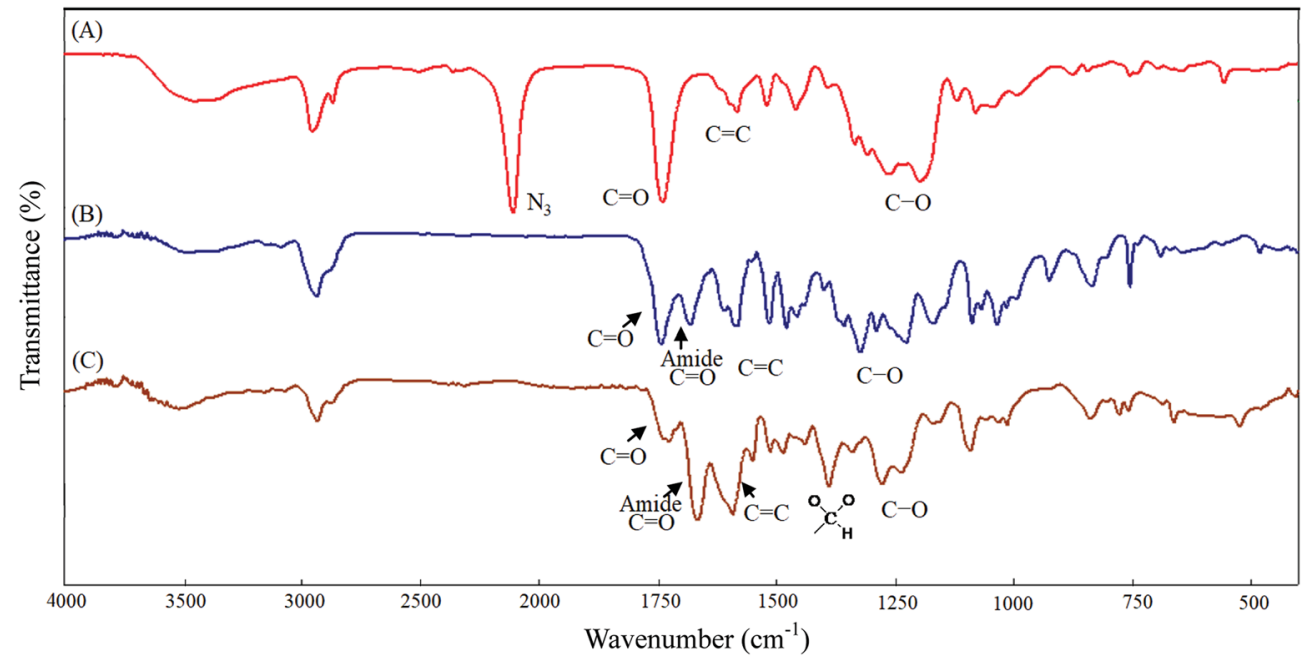

Fig. 2 IR spectra of (A) HONB-P( $\left.\alpha \mathrm{N}_{3} \mathrm{CL}\right)_{10}$, (B) HONB-P( $\left.\alpha \mathrm{N}_{3} \mathrm{CL}-\mathrm{g}-\mathrm{PONBIMC}\right)_{10}$, (C) Gluco-ONB-P( $\left.\alpha \mathrm{N}_{3} \mathrm{CL}-\mathrm{g}-\mathrm{PONBIMC}\right)_{10}$. 


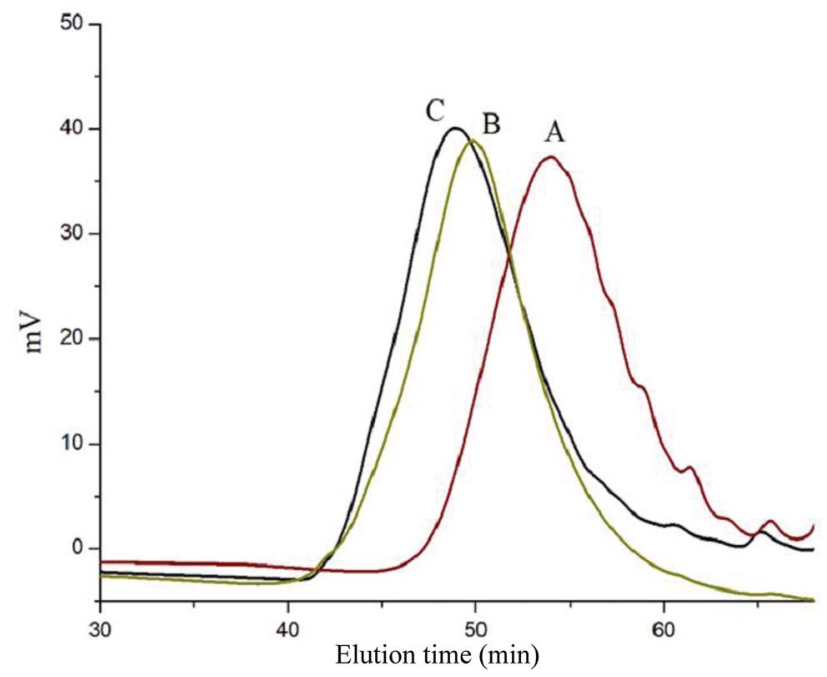

Fig. 3 GPC curve of (A) HONB-P( $\left.\alpha \mathrm{N}_{3} \mathrm{CL}\right)_{10}$, (B) HONB-P $\left(\alpha \mathrm{N}_{3} \mathrm{CL}-g\right.$ PONBIMC $)_{10}$, and (C) Gluco-ONB-P( $\left.\alpha N_{3} C L-g-P O N B I M C\right)_{10}$.

Prepare of 5-propargyoxy-2-nitrobenzyl indomethacinate (PONBIMC). 2-Nitro-5-propargyoxybenzyl alcohol ${ }^{27}$ (1.06 g, 5.10 $\mathrm{mmol})$ and IMC $(2.19 \mathrm{~g}, 6.12 \mathrm{mmol})$ was dissolved in THF (15 $\mathrm{mL}$ ). Under $\mathrm{N}_{2}$ atmosphere, the DMAP (73 $\mathrm{mg}, 0.61 \mathrm{mmol}$ ) and DCC ( $3.2 \mathrm{~g}, 15.3 \mathrm{mmol})$ were added. The reaction mixture was stirred overnight at room temperature. Then, the mixture was filtered and concentrated in vacuo. The residue was purified by flash column chromatography with hexane/EA $(5: 1$ to $2: 1)$ to give 5-propargyoxy-2-nitrobenzyl indomethacinate $(4.70 \mathrm{~g}, 86 \%$ yield). ${ }^{1} \mathrm{H} \mathrm{NMR}\left(\mathrm{CDCl}_{3}, \delta\right): 8.18(\mathrm{~d}, 1 \mathrm{H}), 7.79(\mathrm{~d}, 2 \mathrm{H}) .7 .49(\mathrm{~d}, 2 \mathrm{H})$, $7.0(\mathrm{~d}, 1 \mathrm{H}), 6.98(\mathrm{dd}, 1 \mathrm{H}), 6.89(\mathrm{~d}, 1 \mathrm{H}), 6.86(\mathrm{~d}, 1 \mathrm{H}), 6.68(\mathrm{~d}, 1 \mathrm{H})$, $5.59(\mathrm{~s}, 2 \mathrm{H}), 4.40(\mathrm{~s}, 2 \mathrm{H}), 3.84(\mathrm{~s}, 6 \mathrm{H}), 2.45(\mathrm{~s}, 1 \mathrm{H})$.

\section{Synthesis of HONB-P $\left(\alpha N_{3} C L\right)_{n}$ polymers}

All glassware was dried in an oven and handled under a dry nitrogen stream. 5-Hydroxy-2-nitrobenzyl alcohol (HONB, $275.1 \mathrm{mg}, 1.63 \mathrm{mmol}$ ), as an initiator, and $\alpha \mathrm{ClCL}$ (2.41 g, 16.27 $\mathrm{mmol}$ ) were introduced into a flask, heated under a dry nitrogen stream, and dissolved in $50 \mathrm{~mL}$ of toluene. Subsequently, SnOct $_{2}$ (36 mg, $1.5 \mathrm{wt} \%$, based on the weight of HONB and aClCL) was added to the flask. The flask was purged with nitrogen and refluxed for $8 \mathrm{~h}$, and the solution was vacuum- concentrated under reduced pressure. The resulting product (HONB-P $(\alpha \mathrm{ClCL})_{10}$ ) was dissolved in $\mathrm{CHCl}_{3}$, and precipitated into excess $n$-hexane/diethyl ether $(5: 1 \mathrm{v} / \mathrm{v})$ with stirring. The purified polymer was dried in vacuo at $50{ }^{\circ} \mathrm{C}$ for $24 \mathrm{~h}$ and analyzed subsequently. Yield: $85 \%$. ${ }^{1} \mathrm{H}$ NMR (400 MHz, $\mathrm{CDCl}_{3}$ ) $\delta$ ppm: 8.18 (d, $\mathrm{Ar} H$ ), 7.04 (d, $\mathrm{Ar} H$ ), 6.88 (dd, $\mathrm{Ar} H$ ), 5.60 (s, benzyl $\mathrm{CH}_{2}{ }^{-}$), 4.25 $\left(\mathrm{t},-\mathrm{C}_{\alpha} \mathrm{HCl}^{-}\right), 4.21\left(\mathrm{t},-\mathrm{CH}_{2} \mathrm{O}-\right), 1.91-2.15\left(\mathrm{~m},-\mathrm{C}_{\beta} \mathrm{H}_{2}-\right), 1.74$ $\left(\mathrm{m},-\mathrm{C}_{\delta} H_{2}-\right)$, and $1.40-1.66\left(\mathrm{~m},-\mathrm{C}_{\gamma} H_{2}-\right)$. Subsequently, the chloro was substituted by the azide. HONB-P $(\alpha \mathrm{ClCL})_{10}(2.68 \mathrm{~g}$, $1.28 \mathrm{mmol}$, molar mass $=2090 \mathrm{~g} \mathrm{~mol}^{-1}$ ) was dissolved in $10 \mathrm{~mL}$ of dry DMF, followed by the addition of excess $\mathrm{NaN}_{3}(1.01 \mathrm{~g}, 15.53$ $\mathrm{mmol})$. The mixture was stirred at room temperature for $24 \mathrm{~h}$, the solvent was completely removed through rotary evaporation under reduced pressure. The crude product was subsequently dissolved in $\mathrm{CH}_{2} \mathrm{Cl}_{2}$, and the insoluble salt was removed through filtration. After concentration, the modified polymer was precipitated in excess cold diethyl ether. The copolymer HONB$\mathrm{P}\left(\alpha \mathrm{N}_{3} \mathrm{CL}\right)_{10}$ was obtained at a $91 \%$ yield. Fig. $1 \mathrm{~A}$ and $2 \mathrm{~A}$ depict the representative ${ }^{1} \mathrm{H}$ NMR and infrared (IR) spectra of HONB$\mathrm{P}\left(\alpha \mathrm{N}_{3} \mathrm{CL}\right)_{10}$, respectively.

\section{Synthesis of HONB-P $\left(\alpha N_{3} \mathrm{CL}-g \text {-alkyne }\right)_{n}$ block-grafted polymer with pendant pyrene or IMC groups}

HONB-P $\left(\alpha \mathrm{N}_{3} \mathrm{CL} \text { - } g \text {-alkyne }\right)_{n}$ was prepared through coppercatalyzed azide-alkyne cycloaddition. $\mathrm{HONB}-\mathrm{P}\left(\alpha \mathrm{N}_{3} \mathrm{CL}\right)_{n}(1.10$ $\mathrm{g}$ ) and various types of alkynes (such as 1-hexyne, 1-decayne, 2propargyoxymethyl pyrene, and 5-propargyoxy-2-nitrobenzyl indomethacinate) were dissolved in THF $(10 \mathrm{~mL})$. Subsequently, CuI (0.01 eq.), and $\mathrm{Et}_{3} \mathrm{~N}$ (0.1 eq.) were added under a nitrogen atmosphere. After one freeze pump thaw cycle, the click reaction was conducted at room temperature for $24 \mathrm{~h}$ and stopped by exposure to air. Copper ions were removed from the polymer solution by using a short alumina column. The polymer was purified by precipitating into an excess of cold diethyl ether and drying under reduced pressure. The yield of the copolymers was $83-92 \%$. Fig. $1 \mathrm{C}$ and $2 \mathrm{~B}$ depict the representative ${ }^{1} \mathrm{H}$ NMR and IR spectra of $\mathrm{HONB}-\mathrm{P}\left(\alpha \mathrm{N}_{3} \mathrm{CL}-\mathrm{g} \text {-PONBIMC }\right)_{10}$, respectively.

\section{Synthesis of Glycol-ONB-P( $\left(\alpha \mathrm{N}_{3} \mathrm{CL}-g \text {-alkyne }\right)_{n}$ block-grafted polymers and prodrug}

We used a typical procedure for coupling 3-bromopropyl-sugar with HONB-P $\left(\alpha \mathrm{N}_{3} \mathrm{CL}-g \text {-alkyne }\right)_{n}$. First, a mixture of HONB-

Table 1 Results of the coupling of HONB-P $\left(\alpha N_{3} \mathrm{CL} \text {-g-alkyne/or drug }\right)_{n}$ with Glyco $\left(\mathrm{CH}_{2}\right)_{3} \mathrm{Br}$

\begin{tabular}{|c|c|c|c|c|c|}
\hline Polymer $^{a}$ & $M_{\mathrm{n}, \mathrm{th}}^{b}$ & $M_{\mathrm{n}, \mathrm{NMR}}{ }^{c}$ & $M_{\mathrm{n}, \mathrm{GPC}}{ }^{d}$ & $M_{\mathrm{w}} / M_{\mathrm{n}}(\mathrm{PDI})^{d}$ & Isolated yield (\%) \\
\hline Gluco-ONB-P $\left(\alpha \mathrm{N}_{3} \mathrm{CL}-g \text {-Hexy }\right)_{18}$ & 4658 & 7735 & 8580 & 1.29 & 91 \\
\hline Gluco-ONB-P $\left(\alpha \mathrm{N}_{3} \text { CL- } g \text {-Decy }\right)_{17}$ & 5370 & 8940 & 10450 & 1.79 & 69 \\
\hline Gluco-ONB-P $\left(\alpha \mathrm{N}_{3} \text { CL- } g \text {-Ppyren }\right)_{12}$ & 5489 & 6236 & 6100 & 1.17 & 56 \\
\hline Gluco-ONB-P $\left(\alpha \mathrm{N}_{3} \mathrm{CL}-g\right.$-Ppyren $\left.2 /-\mathrm{Hexy}_{24}\right)$ & 6968 & 7080 & 7910 & 1.35 & 76 \\
\hline Malto-ONB-P $\left(\alpha \mathrm{N}_{3} \mathrm{CL}-g\right.$-Ppyren $/$--Hexy 24$)$ & 7131 & 6948 & 7510 & 1.08 & 86 \\
\hline Gluco-ONB-P $\left(\alpha \mathrm{N}_{3} \mathrm{CL}-g \text {-PONBIMC }\right)_{10}$ & 7400 & 6788 & 6280 & 1.40 & 83 \\
\hline
\end{tabular}

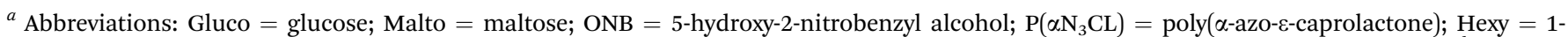

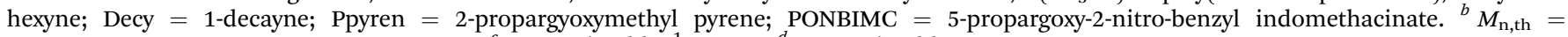

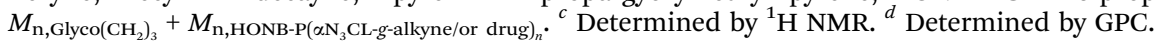


$\mathrm{P}\left(\alpha \mathrm{N}_{3} \mathrm{CL} \text {-g-alkyne }\right)_{n} \quad(0.17 \mathrm{mmol})$ and potassium carbonate (71.6 mg, $0.52 \mathrm{mmol}$ ) was stirred in DMF $(5 \mathrm{~mL})$ for $1 \mathrm{~h}$ at $60{ }^{\circ} \mathrm{C}$. 3-Bromopropyl D-glucopyranoside $(63.2 \mathrm{mg}, 0.21 \mathrm{mmol})$ in DMF ( $3 \mathrm{~mL}$ ) was subsequently added, and the mixture was stirred at $60{ }^{\circ} \mathrm{C}$ for $24 \mathrm{~h}$. DMF was removed under reduced pressure. The residue was dissolved in $\mathrm{CHCl}_{3}$, and precipitated into an excess of cold diethyl ether while stirring. The obtained solids were further purified through dialysis [cellulose membrane, molecular weight cutoff (MWCO): $3500 \mathrm{Da}$ ] against $\mathrm{CHCl}_{3}$ for $24 \mathrm{~h}$. The purified polymers Glyco-ONB-P $\left(\alpha \mathrm{N}_{3} \mathrm{CL}-g \text {-alkyne }\right)_{n}$ were dried in vacuo at $60{ }^{\circ} \mathrm{C}$ for $24 \mathrm{~h}$ to be obtained in $56-91 \%$ yield and analyzed. Fig. 1D and 2C depict the representative ${ }^{1} \mathrm{H}$ NMR and IR spectra of Gluco-ONB-P $\left(\alpha \mathrm{N}_{3} \mathrm{CL}-g \text {-PONBIMC }\right)_{10}$, respectively.

\section{Ultraviolet irradiation}

Glyco-ONB-P $\left(\alpha \mathrm{N}_{3} \mathrm{CL} \text { - } g \text {-alkyne }\right)_{n}(1.5 \mathrm{mg})$ in $1 \mathrm{~mL}$ of inhibitorfree THF or polymer micelles in phosphate buffer solution (PBS) (0.01 M, pH 7.4) were exposed to ultraviolet (UV) irradiation sourced from a UV light system (model PR-2000, Phnchum Co., Taiwan), equipped with a Hitachi FL8BL-B lamp (352 nm, $8 \mathrm{~W} \times 8 \mathrm{~W}$ ). To prevent UV absorption, the samples were placed in quartz cuvettes with a spot area of approximately $1 \mathrm{~cm}^{2}$ and irradiated at room temperature for an assigned duration. Radiation was applied vertically from the top of the cuvette.

\section{Preparation of polymeric micelles}

Polymeric micelles of Glyco-ONB-P $\left(\alpha \mathrm{N}_{3} \mathrm{CL}-g \text {-alkyne }\right)_{n}$ were prepared through dialysis. Briefly, a solution of Glyco-ONB$\mathrm{P}\left(\alpha \mathrm{N}_{3} \mathrm{CL} \text { - } g \text {-alkyne }\right)_{n}$ (30 mg) in $5 \mathrm{~mL}$ of DMF was transferred in a dialysis bag with a MWCO of 3500 Da. The solution was dialyzed against deionized water at an ambient temperature for $24 \mathrm{~h}$. The water was replaced at $2 \mathrm{~h}$ intervals.

\section{Determination of drug loading content and drug entrapment efficiency}

Glyco-ONB-P $\left(\alpha \mathrm{N}_{3} \mathrm{CL} \text { - } g \text {-alkyne }\right)_{n}$ [50-fold critical micelle concentration (CMC) value] was dissolved in $6 \mathrm{~mL}$ of methylene chloride using the oil-in-water evaporation technique. IMC, the antiinflammatory drug, served as a model drug and was added to the polymer at a $1: 1$ weight ratio. The solution was added dropwise to $150 \mathrm{~mL}$ of distilled water containing $1 \mathrm{wt} \%$ polyvinyl alcohol and stirred vigorously. Polyvinyl alcohol acted as a surfactant and reduced micellar aggregation. The mixture was sonicated for $1 \mathrm{~h}$ at an ambient temperature to reduce the droplet size. The resulting emulsion was stirred at an ambient temperature overnight to evaporate the methylene chloride. The unloaded IMC residue was removed through filtration using a Teflon filter (Whatman) with an average pore size of $0.45 \mu \mathrm{m}$. The micelles were obtained through vacuum drying. Later, a weighed amount of micelles was disrupted by adding a 10 -fold excess volume of DMF. The drug content was assayed spectrophotometrically at $320 \mathrm{~nm}$ using a diode array UV-visible spectrophotometer. The following equations were used to calculate the drug loading content (DLC) and drug entrapment efficiency (DEE):
(A)

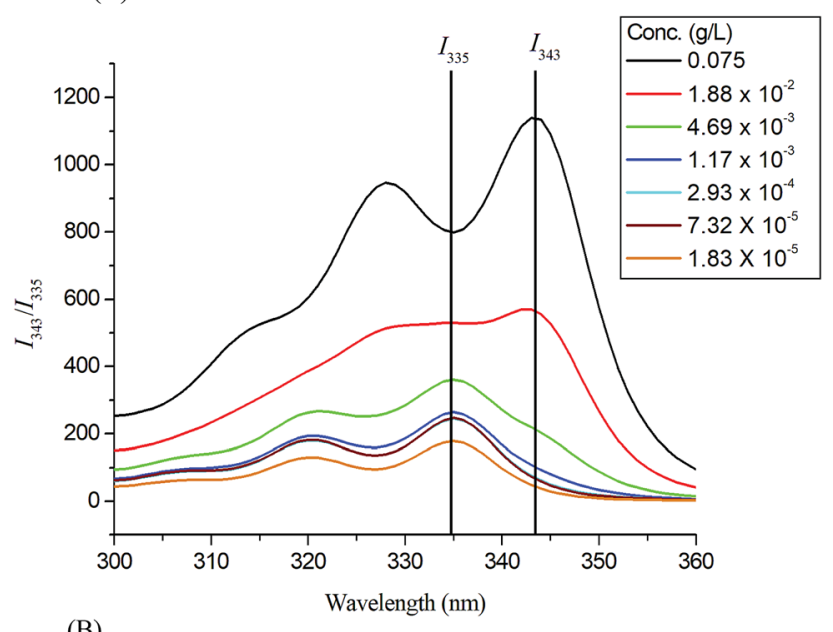

(B)

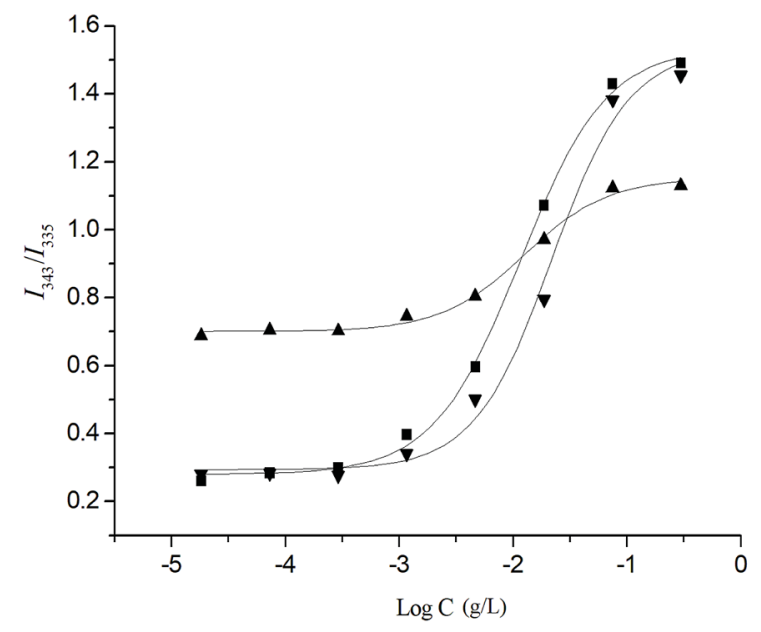

Fig. 4 (A) Excitation spectra of pyrene Gluco-ONB-P $\left(\alpha N_{3} C L-g\right.$ Ppyren $)_{12}$ micelles monitored at $\lambda_{\mathrm{em}}=390 \mathrm{~nm}$ with different concentrations. (B) Plot of the $/ 343 / /_{335}$ intensity ratio (from pyrene excitation spectra: pyrene concentration $=6.1 \times 10^{-7} \mathrm{M}$ ) versus the logarithm of the concentration (log C) of Glyco-ONB-P $\left(\alpha N_{3} C L-g\right.$ -

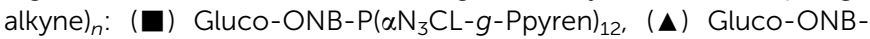
$\mathrm{P}\left(\alpha \mathrm{N}_{3} \mathrm{CL}-\mathrm{g}\right.$-Ppyren $\left.2 /-\mathrm{Hexy}_{24}\right),(\boldsymbol{\nabla})$ Malto-ONB-P( $\alpha \mathrm{N}_{3} \mathrm{CL}-g-$ Ppyren $_{2} /-$ $\left.\mathrm{Hexy}_{24}\right)$.

\section{$\operatorname{DLC}(\%)=$ \\ (weight of the drug in micelles/weight of micelles) $\times 100$}

$\operatorname{DEE}(\%)=$

(weight of the drug in micelles/

weight of the drug provided initially) $\times 100$

\section{Analysis of in vitro drug release}

Appropriate amounts of IMC-incorporated or conjugated micelles $(110.2 \mathrm{mg})$ were weighed and suspended in $10 \mathrm{~mL}$ of PBS (0.01 M, pH 7.4). The micellar solution was transferred to a dialysis membrane bag $(\mathrm{MWCO}=3500 \mathrm{Da})$, and the bag was placed in $50 \mathrm{~mL}$ of a PBS release medium. The medium was 
exposed to UV irradiation at $37^{\circ} \mathrm{C}$. At predetermined intervals, $3 \mathrm{~mL}$ aliquots of the aqueous solution were withdrawn from the release medium, and an identical volume of the fresh buffer solution was added. The concentration of the released IMC was determined using a UV-visible spectrophotometer at a wavelength of $320 \mathrm{~nm}$. The rate of controlled drug release was measured on the basis of the cumulatively released weight of the drug by using the calibration curve for the drug.

\section{Carbohydrate-lectin binding recognition}

The lectin recognition activity of the Glyco-ONB-P $\left(\alpha \mathrm{N}_{3} \mathrm{CL}-\mathrm{g}\right.$ alkyne $)_{n}$ solution was analyzed by assessing the change in turbidity at $600 \mathrm{~nm}$ at room temperature. A $2 \mathrm{mg} \mathrm{mL}^{-1}$ sample of concanavalin A (Con A) lectin was prepared in PBS (0.01 M, $\mathrm{pH}$ 7.4). Subsequently, $600 \mu \mathrm{L}$ of the lectin solution was transferred into a cuvette, and the baseline measurement was recorded. A solution of $60 \mu \mathrm{L}$ of Glyco-ONB-P( $\alpha \mathrm{N}_{3} \mathrm{CL}$ - $g$-alkyne $)_{n}$ at two concentrations $\left(0.1\right.$ and $\left.0.2 \mathrm{mg} \mathrm{mL}^{-1}\right)$ in PBS was added to the cuvette containing the lectin solution. The solution in the cuvette was gently mixed using a pipette; thereafter, the absorbance was recorded immediately at $600 \mathrm{~nm}$ every $200 \mathrm{~s}$. Control readings were obtained using lectin Con $\mathrm{A}$ and the PBS buffer solution without Glyco-ONB-P( $\alpha \mathrm{N}_{3} \mathrm{CL}$-g-alkyne $)_{n}$ under the same experimental conditions.

\section{Determination of in vitro cellular viability}

The CellTiter $96 \circledast$ AQueuous One Solution kit was used to determine cellular viability. The assay was conducted according to the manufacturer's instructions with minor modifications. HeLa cells were seeded in a 24 well plate $\left(3 \times 10^{4}\right.$ cells per well $)$ overnight and subsequently treated with various concentrations of polymers (or DMSO vehicles) added to the DMEM/F12 1:1 medium with $1 \%$ FBS in a humidified incubator at $37{ }^{\circ} \mathrm{C}$, supplied with $5 \%$ carbon dioxide. After $48 \mathrm{~h}$, the medium in each well was removed and replaced with $350 \mu \mathrm{L}$ of warm PBS and $35 \mu \mathrm{L}$ of CellTiter $96 \circledast$ AQueuous One Solution. The mixture was incubated at $37{ }^{\circ} \mathrm{C}$ for $4 \mathrm{~h}$. After incubation, $110 \mu \mathrm{L}$ of supernatant was transferred from each well to a 96 well plate and absorbance was measured at $485 \mathrm{~nm}$ using an enzymelinked immunosorbent assay reader (Hidex, Turku, Finland). Each experiment was conducted in triplicate.

\section{Flow cytometric analysis of the uptake of doxorubicin-loaded micelles}

HeLa cells were seeded in $35 \mathrm{~mm}$ dishes $\left(1.5 \times 10^{5}\right.$ cells per dish) and cultured overnight. Subsequently, after UV irradiation or without treated DOX-loaded Gluco-ONB-P $\left(\alpha N_{3}\right.$ CL-gPONBIMC $)_{10}$ micelles and free DOX $\left(447 \mathrm{ng} \mathrm{mL}^{-1}\right)$ dissolved in DMEM/F12 1: 1 medium with 1\% FBS, were added and the cells were incubated for 1,5 , and $60 \mathrm{~min}$. Next, the cells were trypsinized and fixed with $4 \%$ paraformaldehyde for 15 min prior to analysis. A BD FACS-Calibur flow cytometer (equipped with a $488 \mathrm{~nm}$ argon laser) and CellQuest software were used for the analysis. An FL2 channel captured the fluorescence of the DOX. Each experiment was conducted in triplicate. 

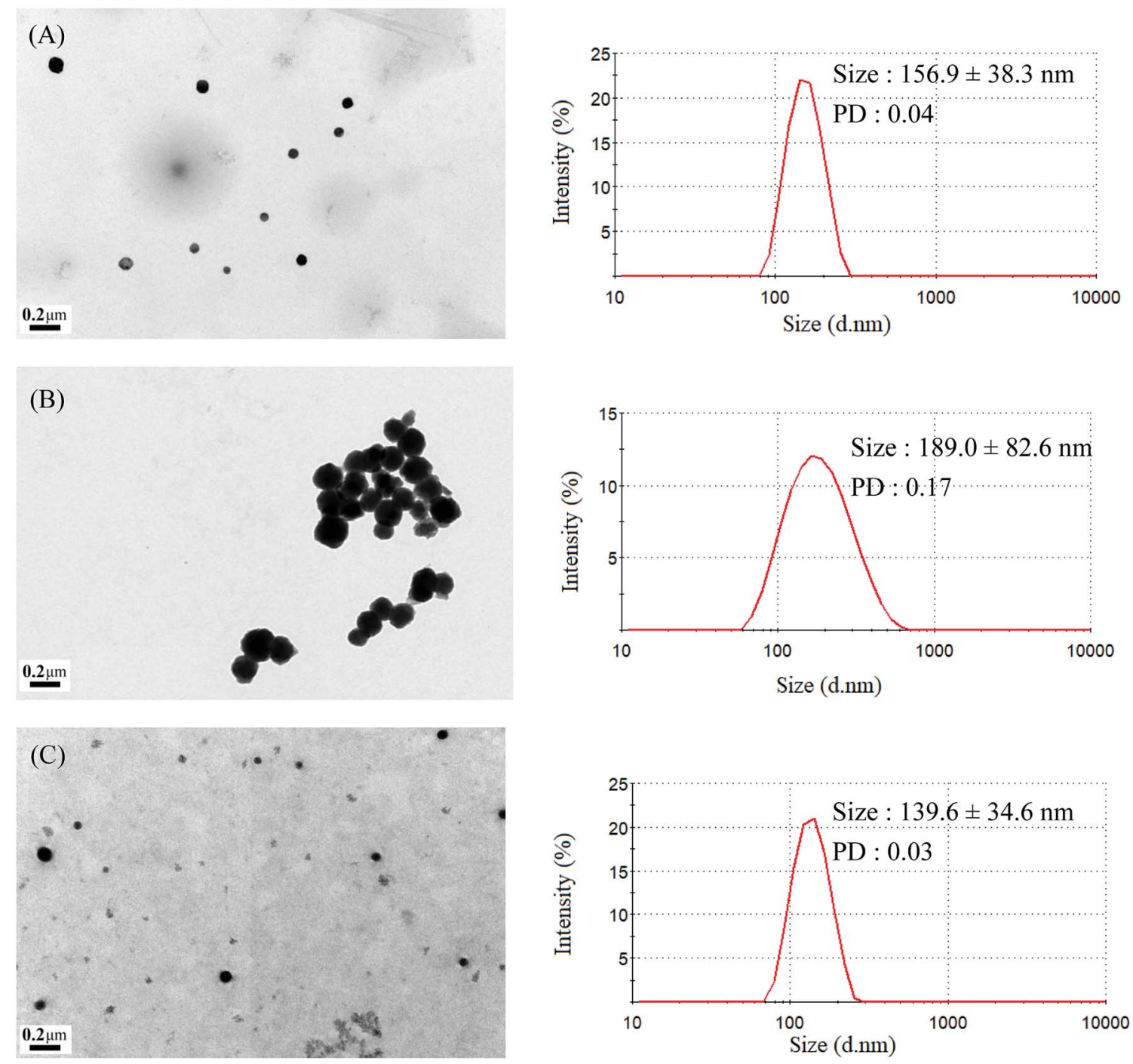

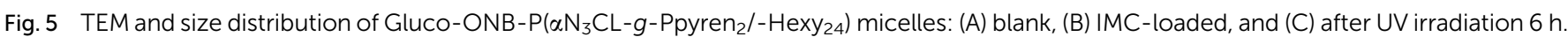

\section{Results and discussion}

\section{Synthesis and characterization of the Glyco-ONB-P $\left(\alpha \mathrm{N}_{3} \mathrm{CL}-\mathrm{g}\right.$ - alkyne) $)_{n}$ block-grafted copolymer}

Scheme 1 depicts the strategy for synthesizing the biorecognizable and photocleavable Glyco-ONB-P $\left(\alpha \mathrm{N}_{3} \mathrm{CL}-g\right.$ alkyne $)_{n}$ copolymer. 5-Hydroxy-2-nitrobenzyl alcohol, a difunctional initiator, which contains two hydroxyl groups, namely benzyl and phenolic hydroxyl groups, was used as a photoresponsive molecule because of its chemical stability and rapid cleavage in response to near-UV irradiation (wavelength $>320$ $\mathrm{nm}) .^{27,28}$ Being more nucleophilic than the phenolic hydroxyl group, the benzyl hydroxyl group initiated the ROP of $\alpha \mathrm{ClCL}$ catalyzed by SnOct $_{2}$. The HONB-P $(\alpha \mathrm{ClCL})_{n}$ polymers with various $\mathrm{P}(\alpha \mathrm{ClCL})_{n}$ lengths were obtained by varying the molar ratio of HONB to $\alpha \mathrm{ClCL}$. The degrees of polymerization $\left(\mathrm{DP}_{\mathrm{n}}\right)$ of $\alpha \mathrm{ClCL}$ can be calculated on the basis of the area ratios of peaks at $4.25 \mathrm{ppm}(\mathrm{CHCl}$ of $\alpha \mathrm{ClCL}$ ) to 5.80 (benzyl protons of HONB). Our calculation result indicates that the average DP of $\alpha \mathrm{ClCL}$ is approximately 10 , and the number-average molecular weight $\left(M_{\mathrm{n}}\right)$ of the polymer is approximately $2 \mathrm{kDa}$. Subsequently, HONB-P $\left(\alpha N_{3} \mathrm{CL}\right)_{10}$ was prepared by a reaction of HONB$\mathrm{P}(\alpha \mathrm{ClCL})_{10}$ and sodium azide. After the reaction, a strong absorption at $2106 \mathrm{~cm}^{-1}$, representing the stretching vibration of azide groups, was observed in the Fourier transform IR (FTIR) spectrum of the polymer (Fig. 2A). The ${ }^{1} \mathrm{H}$ NMR result confirms the conversion of the pendent chlorides to azides. The signal at $4.25 \mathrm{ppm}$ corresponding to the methyne protons next to the chloride groups disappears completely; furthermore, a peak at $3.85 \mathrm{ppm}$ corresponding to the methyne protons next to the azide groups can be observed. Fig. 3A depicts the gel permeation chromatography (GPC) curves of $\operatorname{HONB}-\mathrm{P}\left(\alpha \mathrm{N}_{3} \mathrm{CL}\right)_{10}\left(M_{\mathrm{n}}=\right.$ $\left.2150 \mathrm{~g} \mathrm{~mol}^{-1}, M_{\mathrm{w}} / M_{\mathrm{n}}=1.19\right)$.

The pendant alkyne groups were grafted to $\mathrm{HONB}-\mathrm{P}\left(\alpha \mathrm{N}_{3} \mathrm{CL}\right)_{n}$ backbone through copper-catalyzed azide-alkyne cycloaddition. The FTIR spectrum of HONB-P $\left(\alpha \mathrm{N}_{3} \mathrm{CL}-g \text {-PONBIMC }\right)_{10}$ is shown in Fig. 2B. After the click reaction, the absorbance band at $2106 \mathrm{~cm}^{-1}$ disappeared completely, and new absorbance bands 
(A)

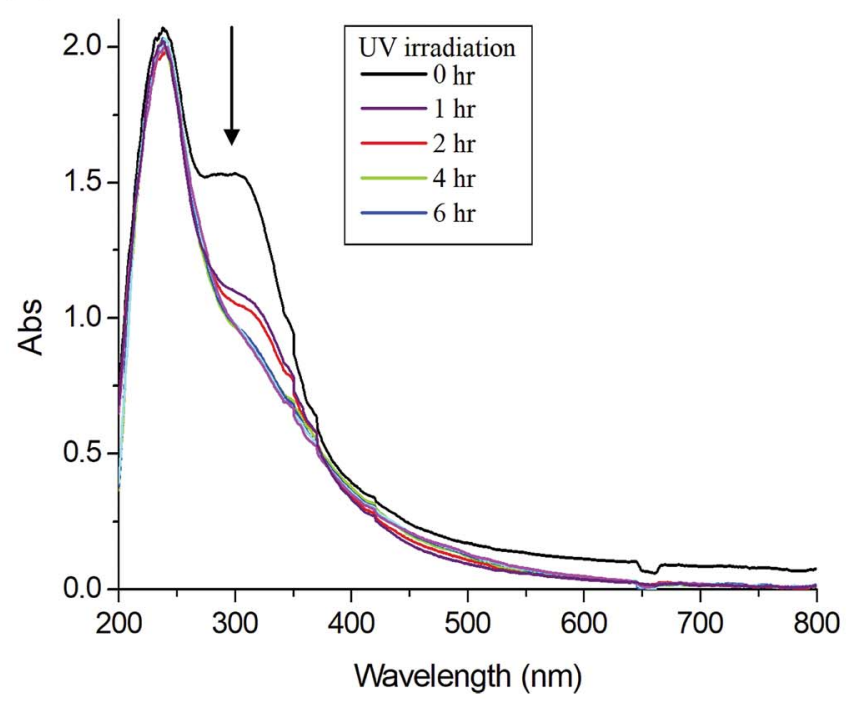

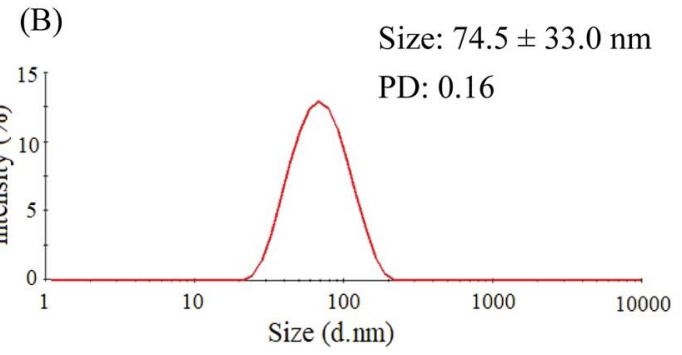

(D)

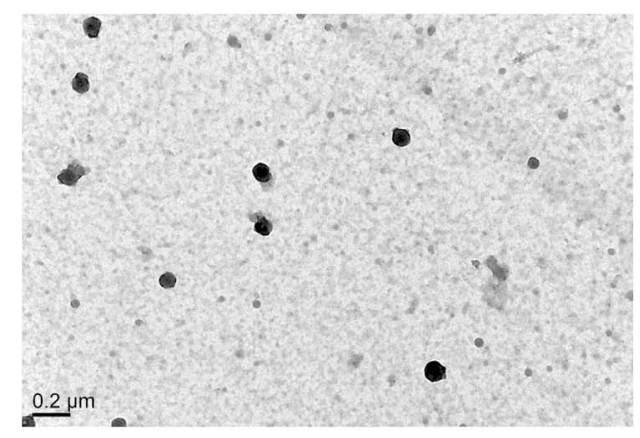

(C)

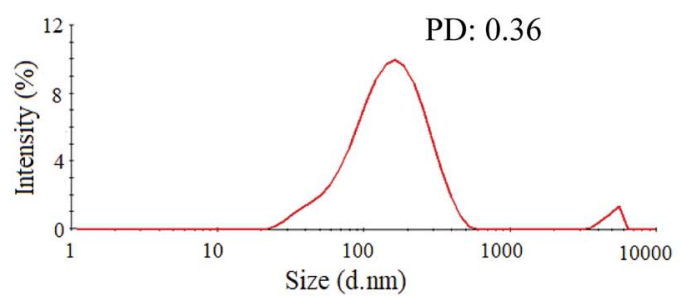

(E)

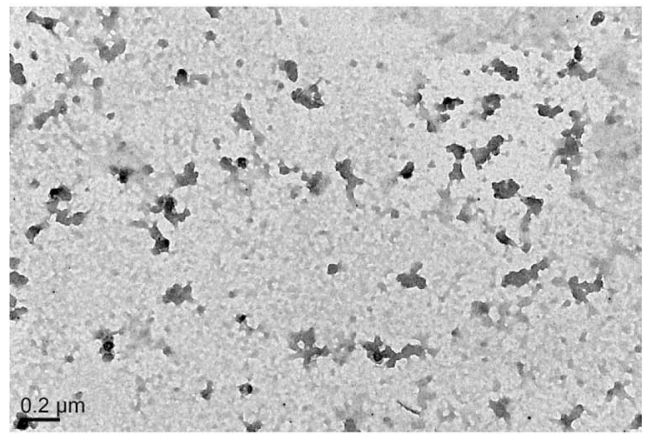

Fig. 6 (A) Time-dependent UV-vis spectra changes of the IMC-conjugate Gluco-ONB-P( $\alpha N_{3} C L$-g-PONBIMC $)_{10}$ micelles treatment under UV irradiation (352 nm, $8 \mathrm{~W} \times 8 \mathrm{~W}$ ), and DLS and TEM changes of Gluco-ONB-P( $\left.\alpha \mathrm{N}_{3} \mathrm{CL}-\mathrm{g}-\mathrm{PONBIMC}\right)_{10}$ micelles: without irradiation (B and D), and with irradiation for $6 \mathrm{~h}$ ( $\mathrm{C}$ and $\mathrm{E})$.

at $1680 \mathrm{~cm}^{-1}$, attributable to the amide carbonyl vibration of the IMC, were observed. The ${ }^{1} \mathrm{H}$ NMR spectra of HONB-P $\left(\alpha \mathrm{N}_{3} \mathrm{CL}-g\right.$ PONBIMC) $)_{10}$ is shown in Fig. 1C. New peaks at 5.30 ppm, corresponding to the methyne protons next to the triazole ring, and at $8.02 \mathrm{ppm}$, corresponding to the vinylic protons of triazole, were observed. Other peaks, including the signals at 7.81, 7.59, $5.61,4.51$, and $3.81 \mathrm{ppm}$ of the IMC, were observed. The GPC trace of HONB-P $\left(\alpha \mathrm{N}_{3} \mathrm{CL}-\mathrm{g} \text {-PONBIMC }\right)_{10}$ is shown in Fig. 3B $\left(M_{\mathrm{n}}\right.$ $=5490 \mathrm{~g} \mathrm{~mol}^{-1}, M_{\mathrm{w}} / M_{\mathrm{n}}=1.43$ ), with unimodal distribution and a shift towards the higher molecular weight region compared with the original HONB-P $\left(\alpha N_{3} \mathrm{CL}\right)_{10}$.
Finally, the phenolic hydroxyl group of the resulting HONB$\mathrm{P}\left(\alpha \mathrm{N}_{3} \mathrm{CL}-g \text {-alkyne }\right)_{n}$ polymer was etherified using Glyco$\left(\mathrm{CH}_{2}\right)_{3} \mathrm{Br}$ (e.g., Gluco- $\left(\mathrm{CH}_{2}\right)_{3} \mathrm{Br}$ and Malto- $\left.\left(\mathrm{CH}_{2}\right)_{3} \mathrm{Br}\right)$ through nucleophilic substitution in DMF at $60{ }^{\circ} \mathrm{C}$ to yield the GlycoONB-P $\left(\alpha \mathrm{N}_{3} \mathrm{CL}-g \text {-alkyne }\right)_{n}$ polymer. Table 1 presents the coupling results. The $M_{\mathrm{n}, \mathrm{GPC}}$ of the block copolymers with different compositions ranged from 6100 to $10450 \mathrm{mg} \mathrm{mol}^{-1}$, and the polydispersity index $\left(M_{\mathrm{w}} / M_{\mathrm{n}}\right)$ ranged from 1.08 to 1.79 . The ${ }^{1} \mathrm{H}$ NMR and FTIR results confirmed the effective coupling of Gluco- $\left(\mathrm{CH}_{2}\right)_{3} \mathrm{Br}$ to yield Gluco-ONB-P $\left(\alpha \mathrm{N}_{3} \mathrm{CL}-g \text {-PONBIMC }\right)_{10}$. The representative ${ }^{1} \mathrm{H}$ NMR spectrum is depicted in Fig. 1D. The 
(A)

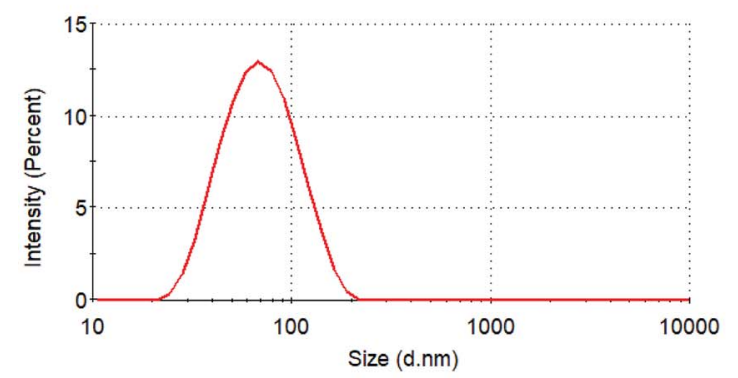

(B)

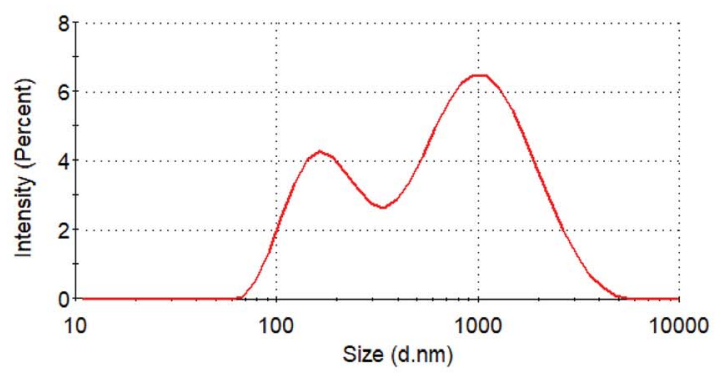

(C)

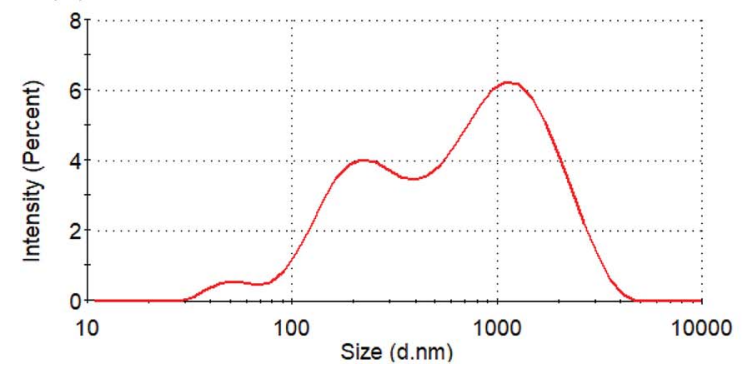

(D)

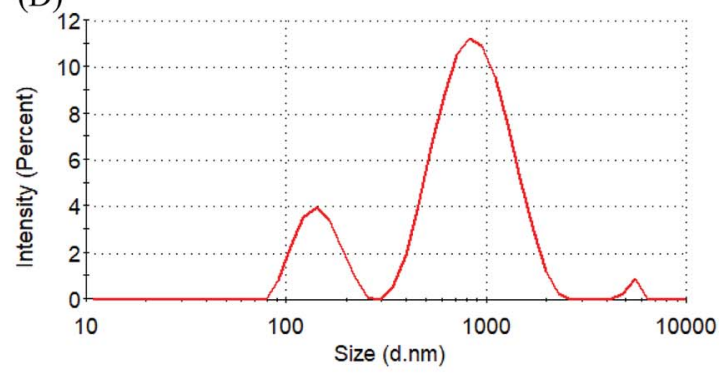

(E)

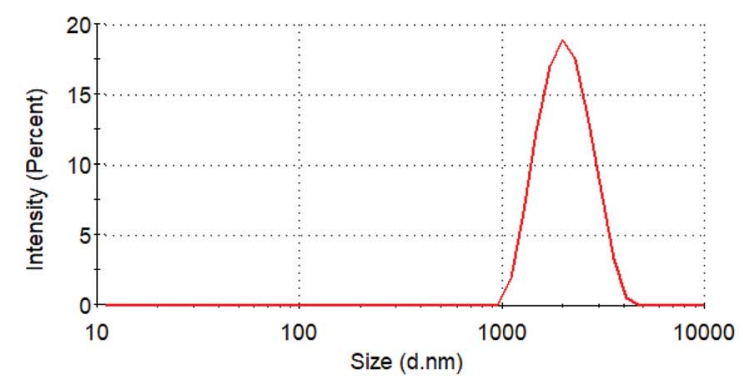

resonance peaks were assigned to the corresponding hydrogen atoms of the Gluco-blocks at $\delta 5.05-5.39$, and 3.21-3.79 ppm. The presence of proton signals from a Gluco-unit suggested successful conjugation. The IR spectrum of Gluco-ONB$\mathrm{P}\left(\alpha \mathrm{N}_{3} \mathrm{CL}-g \text {-PONBIMC }\right)_{10}$ indicated typical carbonyl absorption of an ester of ONB-P $\left(\alpha \mathrm{N}_{3} \mathrm{CL}-g \text {-PONBIMC }\right)_{10}$ at $1720 \mathrm{~cm}^{-1}$, amide carbonyl absorption at $1680 \mathrm{~cm}^{-1}$, hemiacetal C-O absorption at $1400 \mathrm{~cm}^{-1}$, and alcohol C-O absorption at $1250 \mathrm{~cm}^{-1}$ for glucose (Fig. 2C). Fig. 3C depicts the representative GPC curves of Gluco-ONB-P $\left(\alpha \mathrm{N}_{3} \text { CL- } g \text {-PONBIMC }\right)_{10}\left(M_{\mathrm{n}}=6280 \mathrm{~g} \mathrm{~mol}^{-1}, M_{\mathrm{w}} /\right.$ $\left.M_{\mathrm{n}}=1.40\right)$ and depicts a unimodal distribution with a shift in the peak towards the higher molecular weight region, compared with that of the HONB-P $\left(\alpha \mathrm{N}_{3} \mathrm{CL}-\mathrm{g} \text {-PONBIMC }\right)_{10}$.

\section{Glyco-ONB-P $\left(\alpha \mathrm{N}_{3} \mathrm{CL} \text { - } g \text {-alkyne }\right)_{\boldsymbol{n}}$ micelles}

Pyrene molecules encapsulation into the Glyco-ONB-P $\left(\alpha \mathrm{N}_{3} \mathrm{CL}\right.$ $g$-alkyne $)_{n}$ polymers was investigated through fluorescence spectroscopy. The ratio of the intensity of the third emission peak to that of the first emission peak $\left(I_{343} / I_{335}\right)$ was used to characterize the microenvironment of pyrene, with a larger $I_{343} / I_{335}$ indicating a more hydrophobic environment for the pyrene molecules. The fluorescence intensity of the excitation spectrum of pyrene increased with the concentration of the Gluco-ONB-P( $\alpha \mathrm{N}_{3} \mathrm{CL}$-g-Ppyren $)_{12}$ polymer (Fig. $\left.4 \mathrm{~A}\right)$. The characteristic feature of the pyrene excitation spectrum, a red-shift of the $(0,0)$ band from $335 \mathrm{~nm}$ to $343 \mathrm{~nm}$ during partitioning into a micellar hydrophobic core, was used to determine the CMC values of Gluco-ONB-P $\left(\alpha \mathrm{N}_{3} \mathrm{CL}-g \text {-Ppyren }\right)_{12}$. Fig. $4 \mathrm{~B}$ presents the intensity ratios $I_{343} / I_{335}$ of the pyrene excitation spectra and the logarithmic values of the Glyco-ONB-P $\left(\alpha \mathrm{N}_{3} \mathrm{CL}-\right.$ $g$-alkyne $)_{n}$ concentrations. The CMC value was determined according to the interaction between straight-line segments drawn through the points of the lowest polymer concentrations, which formed an almost horizontal line, and the points of the rapidly rising region of the plot. Table 2 lists the CMC values of various Glyco-ONB-P $\left(\alpha \mathrm{N}_{3} \mathrm{CL}-g \text {-alkyne }\right)_{n}$ polymers. The Glyco-ONB-P $\left(\alpha \mathrm{N}_{3} \mathrm{CL} \text {-g-alkyne }\right)_{n}$ polymers formed micelles in the aqueous phase, with the micellar CMC values ranging from 1.9 to $69.8 \mathrm{mg} \mathrm{L}^{-1}$. The Glyco-ONB-P $\left(\alpha \mathrm{N}_{3} \mathrm{CL}-g \text {-alkyne }\right)_{n}$ polymers exhibited lower CMC values than those of the surfactant (e.g., $2.3 \mathrm{~g} \mathrm{~L}^{-1}$ for sodium dodecyl sulfate in water), indicating thermodynamically favorable self-association in Glyco-ONB- 
(A)

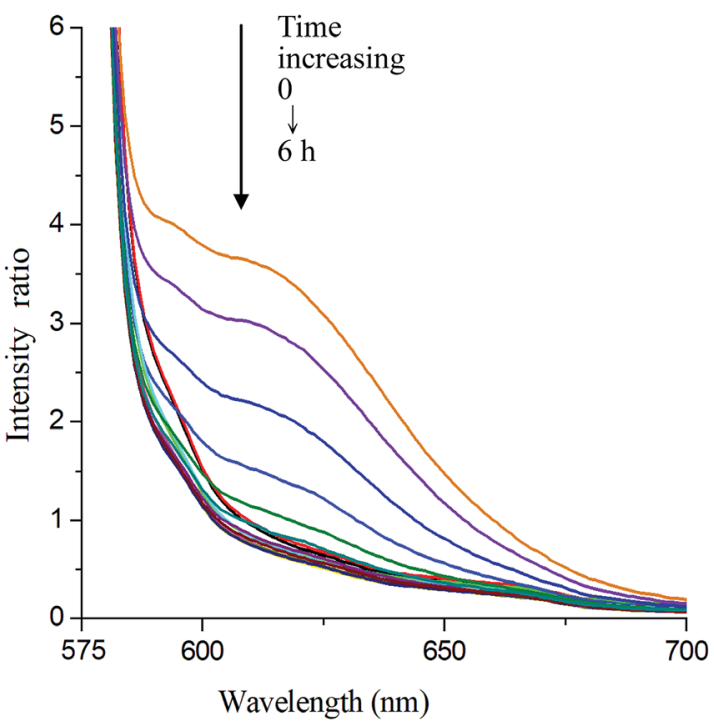

(B)

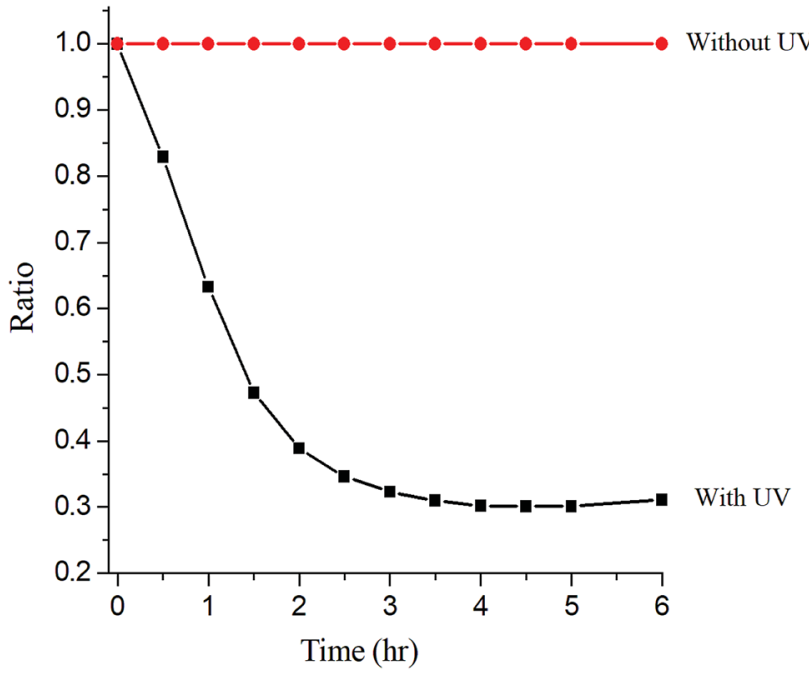

Fig. 8 (A) Fluorescence spectra change of Nile red-loaded GlucoONB-P $\left(\alpha \mathrm{N}_{3} \mathrm{CL}-\mathrm{g}\right.$-Ppyren $/$ - Hexy $\left._{24}\right)$ micelle in PBS $(0.01 \mathrm{M}, \mathrm{pH} 7.4)$ in the presence of UV irradiation $(352 \mathrm{~nm})$ at $25{ }^{\circ} \mathrm{C}$, (B) normalized fluorescence emission intensity $v s$. time of irradiation.

$\mathrm{P}\left(\alpha \mathrm{N}_{3} \mathrm{CL} \text { - } g \text {-alkyne }\right)_{n}$ polymers. The CMC values decreased as the hydrophobicity of the hydrophobic segment increased. When the hydrophobic pyrene or IMC molecules were grafted onto the $\mathrm{P}\left(\alpha \mathrm{N}_{3} \mathrm{CL}\right)_{n}$, the CMC values decreased significantly.

The mean hydrodynamic diameters of micelles ranged from 74.5 to $157.2 \mathrm{~nm}$, with a narrow distribution, and demonstrated a particle size distribution $(\mathrm{PD})$ of $\leq 0.25$. The TEM and size distribution of Gluco-ONB-P $\left(\alpha \mathrm{N}_{3} \mathrm{CL}-\mathrm{g}\right.$-Ppyren $\left.{ }_{2} / \mathrm{Hexy}_{24}\right)$ micelles are demonstrated in Fig. 5A. The spherical morphology was observed. When the drug was incorporated, the micellar size increased (Fig. 5B). The size of an IMC-incorporated GlucoONB-P $\left(\alpha \mathrm{N}_{3} \mathrm{CL}-g\right.$-Ppyren $\left.{ }_{2} / \mathrm{Hexy}_{24}\right)$ micelle was $189.0 \mathrm{~nm}$ larger
(A)

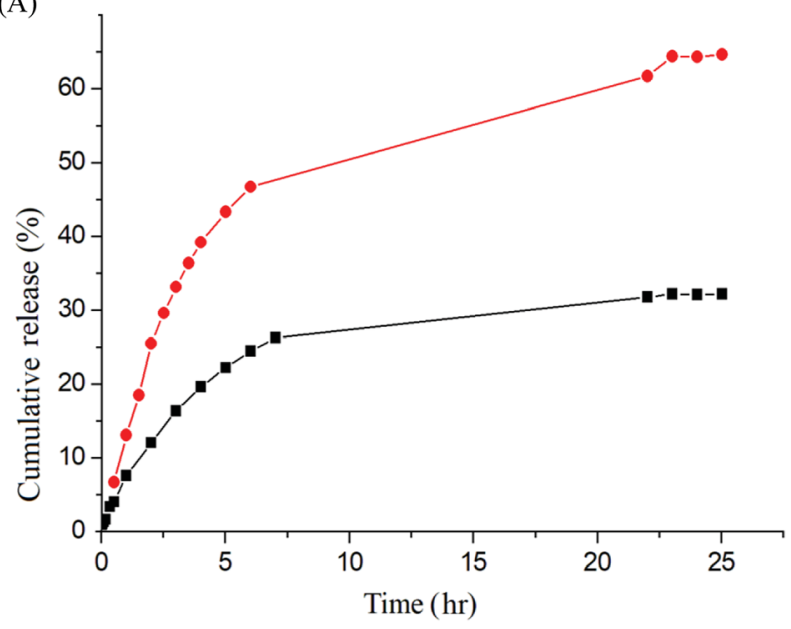

(B)

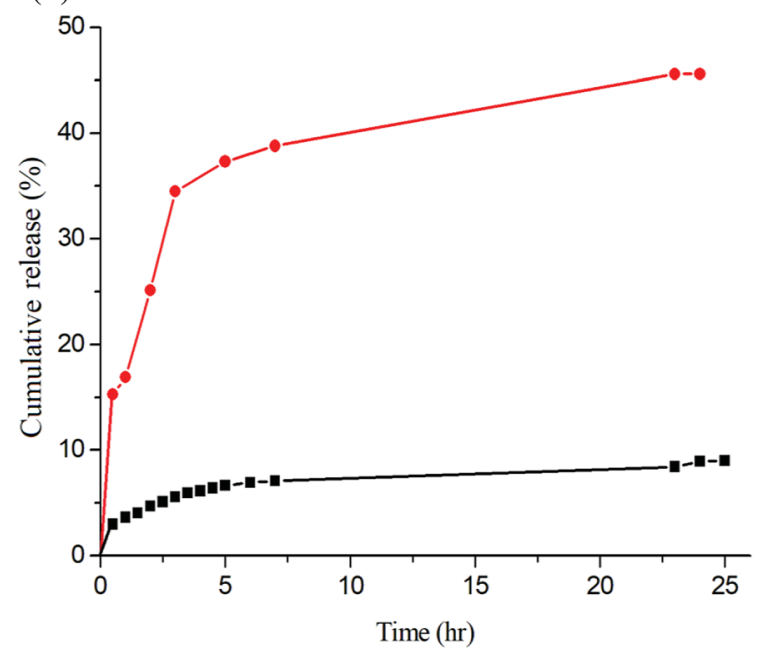

Fig. 9 IMC release (A) the IMC-loaded micelle of Gluco-ONB$\mathrm{P}\left(\alpha \mathrm{N}_{3} \mathrm{CL}-\mathrm{g}\right.$-Ppyren $/$ - - $\left.\mathrm{exy}_{24}\right)$ in the presence of UV irradiation (O) and without irradiation ( $\mathbf{\square}),(B)$ the IMC-conjugate micelle of GlucoONB-P $\left(\alpha \mathrm{N}_{3} \mathrm{CL}-\mathrm{g} \text {-PONBIMC }\right)_{10}$ in the presence of UV irradiation ( and without irradiation ( $\mathbf{\square})$ in PBS $(0.01 \mathrm{M}, \mathrm{pH} 7.4)$ at $37^{\circ} \mathrm{C}$.

than that of a blank micelle (156.9 $\mathrm{nm})$. The increase in micelle size might be attributed to the increase of the hydrophobic core when the IMC is encapsulated into the copolymer, which is in agreement with the thermodynamic aggregation of the block copolymers (BCPs). ${ }^{29}$ However, the micelle size remained $<200 \mathrm{~nm}$ for all formations. A suitable nanoparticle size (diameter: $<200 \mathrm{~nm}$ ) can reduce uptake in the reticulate endothelial system, minimize renal excretion, and increase micelleencapsulated drug accumulation in tumors through increased permeability and retention. ${ }^{30}$ The average diameters measured through DLS were larger than those estimated using through TEM images. This is because DLS reveals the average dimensions of the micelles in aqueous solution, whereas TEM reveals the actual core dimensions of the micelles in a dry state. The TEM and PD of the prodrug Gluco-ONB-P $\left(\alpha \mathrm{N}_{3} \text { CL- } g \text {-PONBIMC }\right)_{10}$ micelles are presented in Fig. $6 \mathrm{~B}$ and $\mathrm{D}$, respectively. 


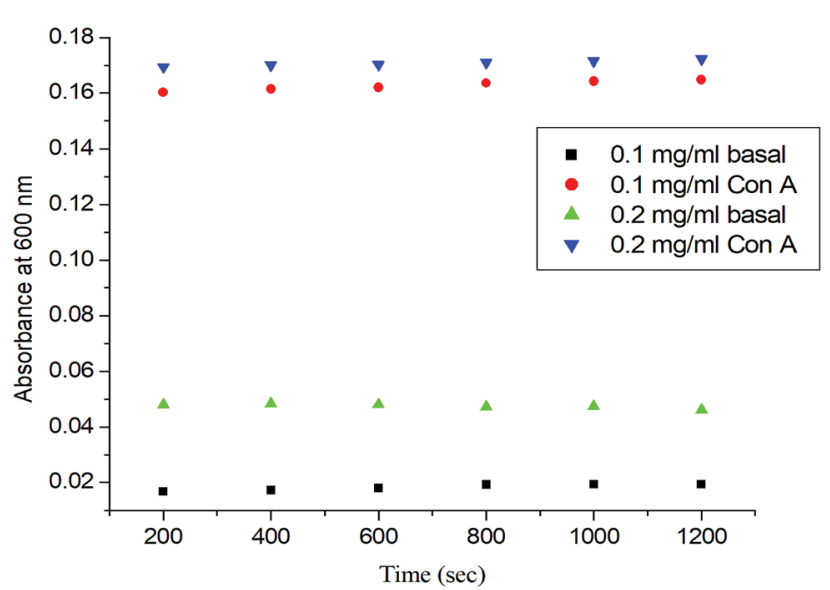

Fig. 10 The absorbance $(450 \mathrm{~nm})$ of the Gluco-ONB-P $\left(\alpha \mathrm{N}_{3} \mathrm{CL}-g\right.$ PONBIMC $)_{10}$ solution upon reaction with lectin Con $A\left(2 \mathrm{mg} \mathrm{mL}^{-1}\right)$ in PBS buffer: concentration $0.2 \mathrm{mg} \mathrm{mL}^{-1}$ with lectin Con $\mathrm{A}(\boldsymbol{\nabla})$ or without lectin Con $\mathrm{A}(\boldsymbol{\Delta})$, concentration $0.1 \mathrm{mg} \mathrm{mL}^{-1}$ with lectin Con $\mathrm{A}$ $(\mathbf{)})$ or without lectin Con $A(\boldsymbol{\square})$

The zeta potential was used to assess micellar stability. When the absolute value of the zeta potential of a micelle was $\geq 30 \mathrm{mV}$, the micelle was stable. ${ }^{31}$ Table 2 indicates that the zeta potential of Glyco-ONB-P $\left(\alpha \mathrm{N}_{3} \mathrm{CL} \text { - } g \text {-alkyne }\right)_{n}$ micelles is in the range of $-20.7 \mathrm{mV}$ to $-41.5 \mathrm{mV}$. The micellar surfaces were negatively charged with comparable zeta potentials. IMC loading lowered the degree of negative charges on the micellar surface because the hydroxyl groups of glycose molecules were protonated by the carboxylic acid group in the IMC molecules. For example, the zeta potential of the IMC-incorporated GlucoONB-P $\left(\alpha \mathrm{N}_{3} \mathrm{CL}-g\right.$-Ppyren $\left.{ }_{2} / \mathrm{Hexy}_{24}\right)$ micelles decreased from $-41.5 \mathrm{mV}$ to $-32.6 \mathrm{mV}$. A less negatively charged surface is favorable for preventing uptake by the phagocytic cells, resulting in faster clearance from blood. ${ }^{32}$ However, to check the stability of the prodrug Gluco-ONB-P $\left(\alpha \mathrm{N}_{3} \mathrm{CL}-g \text {-PONBIMC }\right)_{10}$ micelles in the culture medium, we dispersed the prodrug micelles with $10 \%$ bovine serum albumin (BSA) and incubated them at $37{ }^{\circ} \mathrm{C}$ for 14 day. The stability was studied by DLS measurement and presented in Fig. 7. BSA-treated prodrug micelles exhibited drastic increase in size to about $190 \mathrm{~nm}$ and $1130 \mathrm{~nm}$ with bimodal curve within $30 \mathrm{~min}$. As the incubated time up to 14 day, the size of micelle increased to $2170 \mathrm{~nm}$. These results imply that the prodrug micelle is easily absorption with protein.

\section{Photocleavable behaviors of micelles}

Photo-degradation of micelles was assessed at various irradiation times by monitoring changes in NR fluorescence. ${ }^{33}$ The reduction in the intensity of $\mathrm{NR}$ fluorescence at $610 \mathrm{~nm}$ was recorded. After dissolving the NR and Gluco-ONB-P $\left(\alpha \mathrm{N}_{3} \mathrm{CL}-g\right.$ Ppyren $\left._{2} /-\mathrm{Hexy}_{24}\right)$ in THF $\left(0.5 \mathrm{mg} \mathrm{mL} \mathrm{mL}^{-1}\right.$, NR: polymer ratio $=$ $1: 3)$, water was added to induce micelle formation and concomitant NR encapsulation by the micelle core. THF was subsequently removed through evaporation, and nonsolubilized NR was micro-filtered ( $0.2 \mu \mathrm{m}$ pore filter). The final
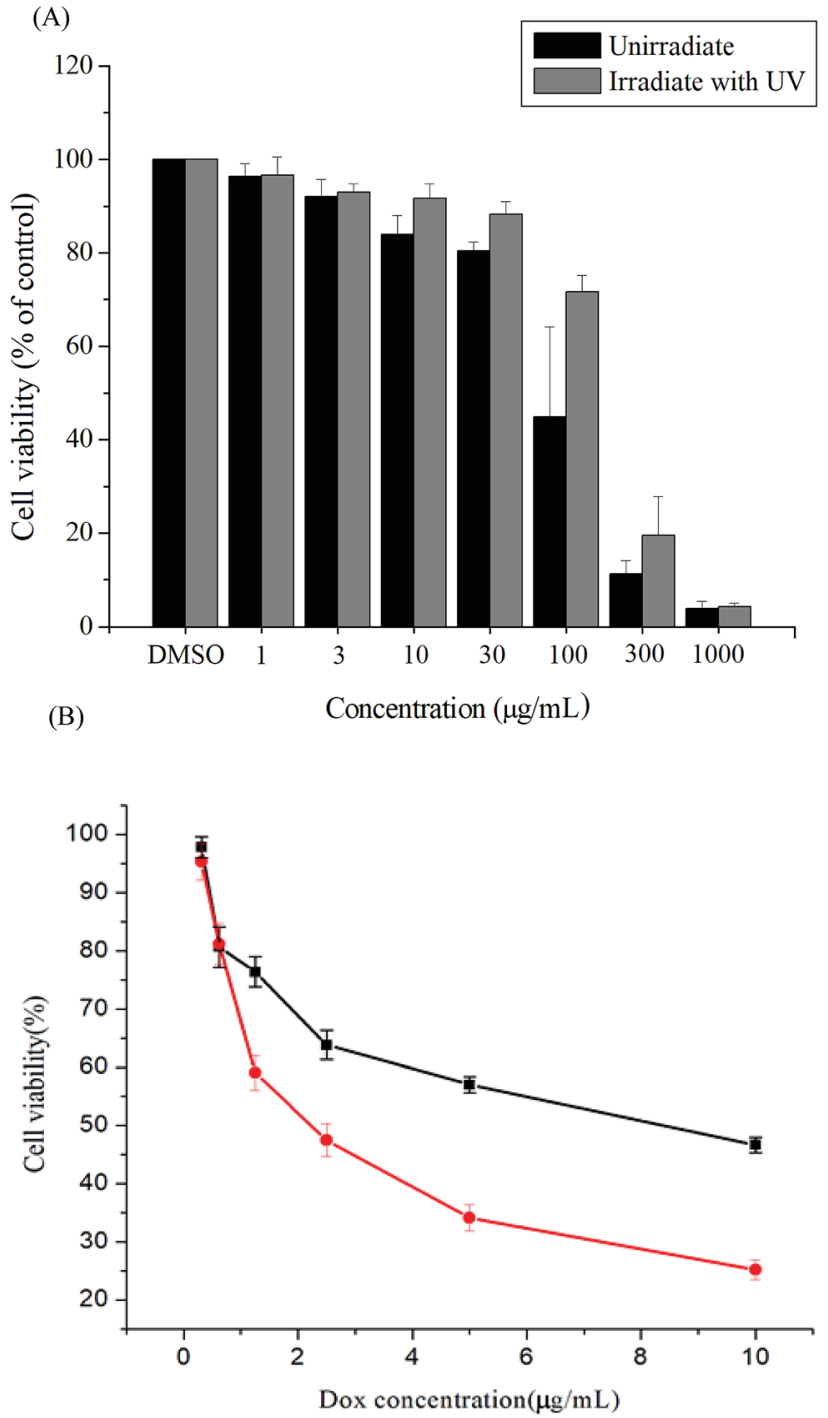

Fig. 11 The cell viabilities of HeLa cells treated: (A) with various concentrations of Gluco-ONB-P( $\alpha \mathrm{N}_{3} \mathrm{CL}-\mathrm{g}$-PONBIMC $)_{10}$ before UV exposure and after $1 \mathrm{~h}$ of UV irradiation, (B) with DOX-loaded GlucoONB-P $\left(\alpha N_{3} C L-g \text {-PONBIMC }\right)_{10}$ micelles $(\boldsymbol{\square})$, and free DOX (0) for 48 h. Data are shown as mean \pm S.E. $(n=3)$.

micellar concentration was adjusted to $0.2 \mathrm{mg} \mathrm{mL}^{-1}$. Fig. 8A depicts the fluorescence emission spectra of NR incorporated in the Gluco-ONB-P $\left(\alpha \mathrm{N}_{3} \mathrm{CL}-\mathrm{g}\right.$-Ppyren $\left.{ }_{2} / \mathrm{Hexy}_{24}\right)$ micelles before and after UV irradiation at various time intervals. Fig. $8 \mathrm{~B}$ plots normalized fluorescence against time; through UV irradiation of the solution, the emission intensity was reduced to $30 \%$ after $6 \mathrm{~h}$ of irradiation. The micelles exhibited photolabile properties in response to light activation. The TEM and PD of Gluco-ONB$\mathrm{P}\left(\alpha \mathrm{N}_{3} \mathrm{CL}-g\right.$-Ppyren $/$ /-Hexy $\left.{ }_{24}\right)$ micelles after $6 \mathrm{~h}$ UV irradiation are presented in Fig. $5 \mathrm{C}$. The results indicated that the destruction of a micelle was not complete because of only one ONB moiety and the steric hindrance of polymer. The photo-degradability of the formed prodrug micelles was subsequently studied. The TEM and size distribution of Gluco-ONB-P $\left(\alpha N_{3}\right.$ CL- $g$ PONBIMC) $)_{10}$ micelles after $6 \mathrm{~h}$ UV irradiation are demonstrated 
(A)

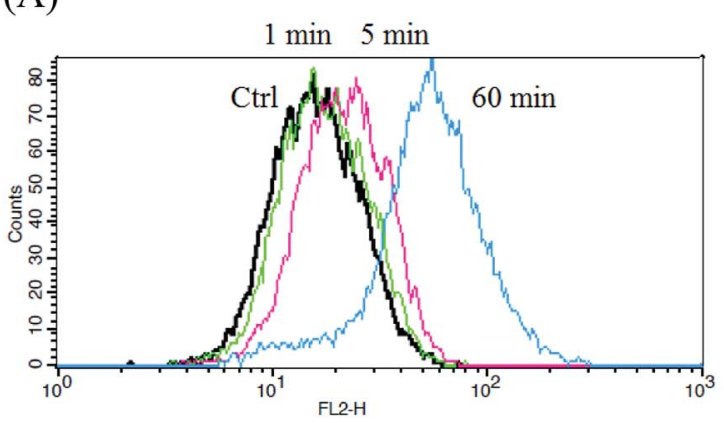

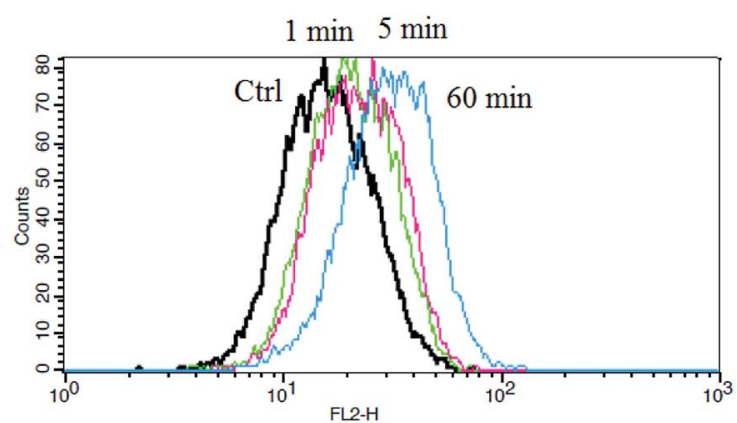

(B)

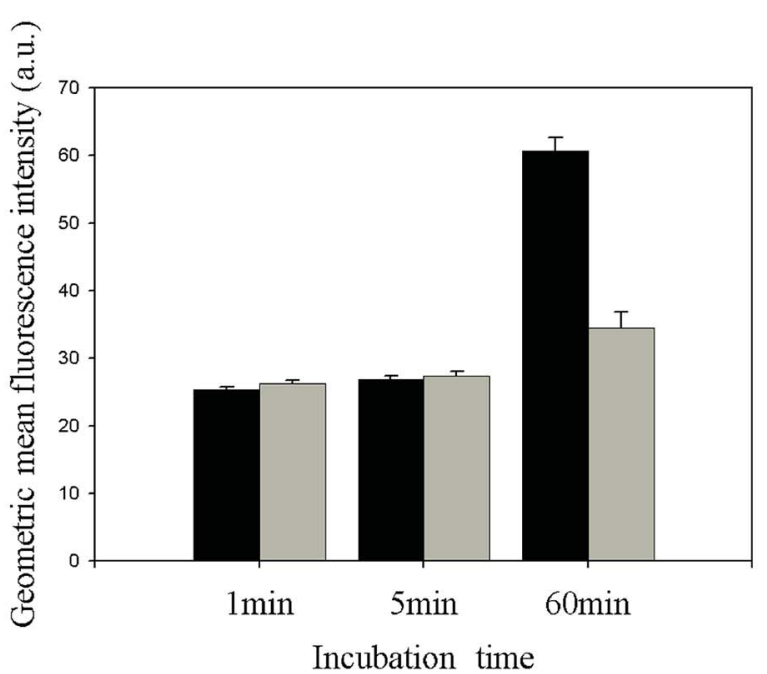

Fig. 12 (A) Flow cytometric histogram profiles of HeLa cells treated with free DOX (left), and DOX-loaded Gluco-ONB-P( $\left.\alpha \mathrm{N}_{3} \mathrm{CL}-\mathrm{g}-\mathrm{PONBIMC}\right)_{10}$ (right) for 1, 5, and $60 \mathrm{~min}$. Control groups were cells that did not receive any treatment, representing basal fluorescent levels, and (B) geometric mean fluorescence intensities of free DOX (black) and DOX-loaded micelles (gray). Data shown mean \pm S. E. $(n=3)$.

in Fig. 6C and E, respectively. Before irradiation, micelles morphologies were uniform. However, after UV irradiation, disintegrated micelles with aggregation were observed, indicating that irradiation for a specific duration induced changes in the assembly state. When expose to UV light, the micelle size of Gluco-ONB-P $\left(\alpha \mathrm{N}_{3} \text { CL- } g \text {-PONBIMC) }\right)_{10}$ increased from 74.5 (PD $=0.16)$ to $163.4 \mathrm{~nm}(\mathrm{PD}=0.36)$, with broader size distribution and larger aggregates with an average diameter $>750 \mathrm{~nm}$. These aggregates are likely fragments of the hydrophobic backbone without Gluco, which arise from the backbone degradation and are insoluble in water. Compared with the photocleavage of Gluco-ONB-P $\left(\alpha \mathrm{N}_{3} \mathrm{CL}-g\right.$-Ppyren $\left.{ }_{2} / \mathrm{Hexy}_{24}\right)$ micelles, the GlucoONB-P $\left(\alpha \mathrm{N}_{3} \mathrm{CL}-g \text {-PONBIMC }\right)_{10}$ micelles demonstrated easier photocleavage because they have greater numbers ONB moieties in the polymer.

\section{Evaluation of drug loading content, entrapment efficiency and in vitro release of IMC}

The DLC and DEE of the polymeric micelles were determined through UV-visible absorption spectroscopy of IMC. IMC, a widely used hydrophobic, nonsteroidal anti-inflammatory drug, was used as a model drug to investigate drug loading in the hydrophobic core. The maximal absorption peak of IMC was proportional to its concentration at $320 \mathrm{~nm}$. After releasing IMC and removing the polymer precipitate, the amount of loaded IMC was determined according to the absorbance at $320 \mathrm{~nm}$. Table 2 lists the calculated drug loading content and entrapment efficiency. At a constant feed weight ratio $\left(W_{\text {IMC }} / W_{\text {copolymer }}\right.$ $=1: 1$ ), the DLC and DEE ranged from $8.21 \%$ to $48.07 \%$ and $16.43 \%$ to $96.14 \%$, respectively, for the Glyco-ONB-P $\left(\alpha \mathrm{N}_{3} \mathrm{CL}-g\right.$ alkyne $)_{n}$ series of polymers. The DLC and DEE increased with the hydrophobicity of the hydrophobic segment. When the longer hydrophobic alkyne or pyrene was grafted onto the hydrophobic block, which exhibits stronger interactions with guest molecules, higher DLC and DEE can therefore be achieved. For the Gluco-ONB-P $\left(\alpha \mathrm{N}_{3} \mathrm{CL}-g \text {-PONBIMC }\right)_{10}$ prodrug, the DLC was calculated approximately $52.71 \%$. Compare with the simple physical encapsulation system, the DLC of the prodrug is high.

The release rate was monitored by determining the concentration of the total amount of released drug. Fig. 9 depicts the release profiles of IMC from the IMC-loaded micelles of GlucoONB-P $\left(\alpha \mathrm{N}_{3} \mathrm{CL}-g\right.$-Ppyren $\left.{ }_{2} / \mathrm{Hexy}_{24}\right)$ and IMC-conjugated micelles of Gluco-ONB-P $\left(\alpha \mathrm{N}_{3} \mathrm{CL}-\mathrm{g} \text {-PONBIMC }\right)_{10}$ prodrug. A biphasic release profile was observed, in which a rapid release stage was followed by a sustained release phase. Compared with the 

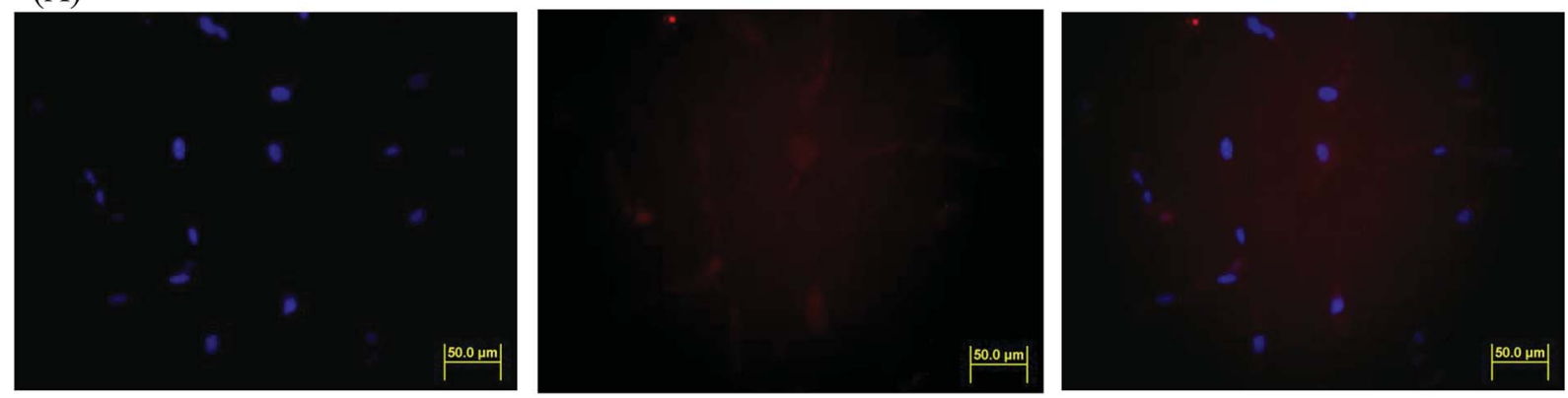

(B)
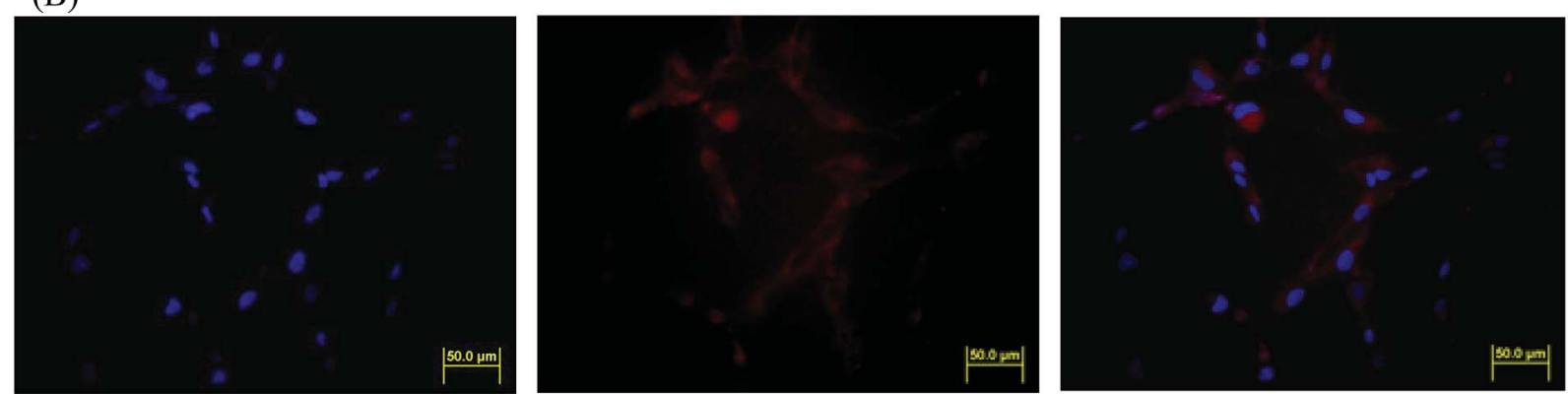

(C)
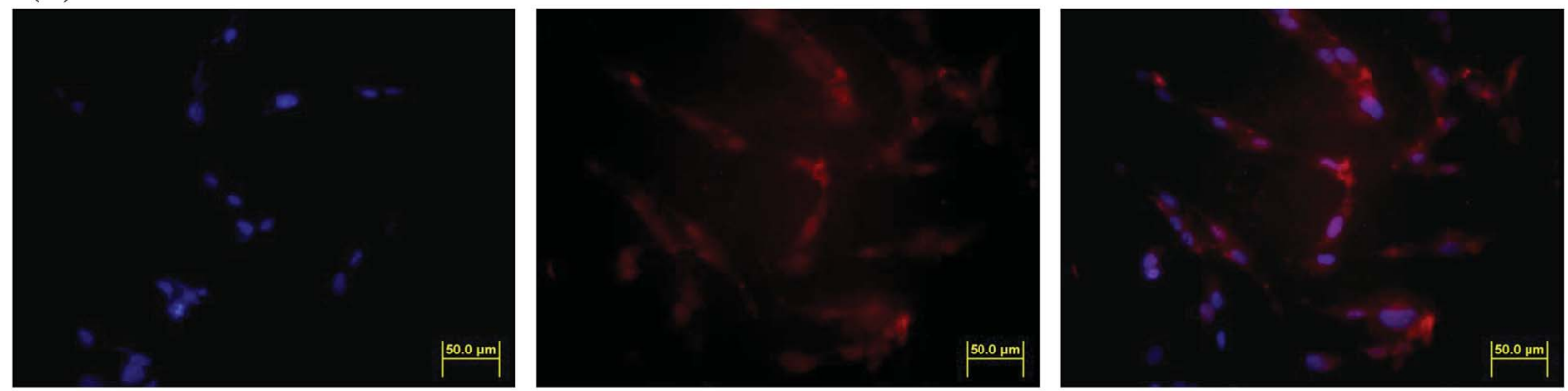

(D)
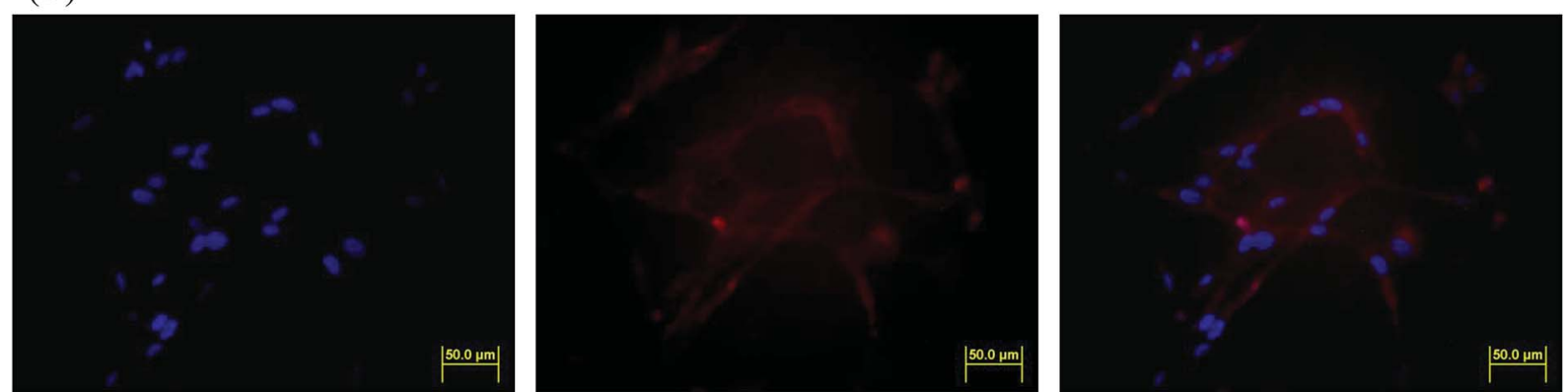

Fig. 13 Fluorescent microscopic images of HeLa cells incubated with free DOX (254.7 ng mL ${ }^{-1}$ ) or DOX-loaded Gluco-ONB-P( $\alpha \mathrm{N}_{3} \mathrm{CL}-\mathrm{g}$ PONBIMC) 10 micelles for different time intervals: (A) free DOX, and (B) DOX-loaded micelles for 1 min; (C) free DOX, and (D) DOX-loaded micelles for $60 \mathrm{~min}$. For each row, images for left to right show the cells with Hoechst 33342 nuclear staining, DOX fluorescence, and the merged image (scale bar $50 \mu \mathrm{m}$; brightness not proportional to fluorescence intensity).

released rates in the absence of UV irradiation, the release rate of IMC was faster during UV irradiation at $37{ }^{\circ} \mathrm{C}$, with approximately $45 \%$ of the encapsulated IMC released in a sustained manner during the first $5 \mathrm{~h}$. After irradiation for $25 \mathrm{~h}$, approximately $65 \%$ of the IMC was released, whereas only $26 \%$ release was exhibited without UV irradiation. For the IMC-conjugated Gluco-ONB-P $\left(\alpha \mathrm{N}_{3} \text { CL- } g \text {-PONBIMC }\right)_{10}$ prodrug system, the release rate of IMC was slower than the IMC-loading micelles of Gluco-ONB-P $\left(\alpha \mathrm{N}_{3}\right.$ CL- $g$-Ppyren $\left.2 /-\mathrm{Hexy}_{24}\right)$. Approximately $45 \%$ of the IMC was released under UV irradiation for $25 \mathrm{~h}$; only $8 \%$ release was observed without irradiation over the same period (Fig. 9B). This can be ascribed to the covalent linkage nature between IMC and polymer backbones within micellar cores. Therefore, those covalent linkages can be cleaved with UV irradiation.

\section{Carbohydrate-lectin binding recognition}

To determine the role of the synthetic glycopolymer, GlycoONB-P $\left(\alpha \mathrm{N}_{3} \mathrm{CL}-g \text {-alkyne }\right)_{n}$, in drug targeting, the ability of the 
(A)

Fig. 14 (A) Flow cytometric histogram profiles of HeLa cells treated with free DOX (left), and after 5 min UV irradiation DOX-loaded Gluco-ONB$\mathrm{P}\left(\alpha \mathrm{N}_{3} \mathrm{CL}-\mathrm{g} \text {-PONBIMC }\right)_{10}$ micelles (right) for 1, 5, and $60 \mathrm{~min}$. Control groups were cells that did not receive any treatment, representing basal fluorescent levels, and (B) geometric mean fluorescence intensities of free DOX (black) and after 5 min UV irradiation DOX-loaded micelles (gray). Data shown mean \pm S. E. $(n=3)$.

synthesized Gluco-ONB-P $\left(\alpha \mathrm{N}_{3} \mathrm{CL}-\mathrm{g} \text {-PONBIMC }\right)_{10}$ polymer to interact with the biological system was assessed. Carbohydrate is essential in several biological recognition events mediated by specific carbohydrate-lectin interactions. Although the exact mechanism of this interaction remains unknown, numerous studies have demonstrated that the mechanism is highly specific and noncovalent. ${ }^{34}$ Thus, an in vitro evaluation of this specific binding event provides an initial test of the ability of a synthetic Gluco-ONB-P $\left(\alpha \mathrm{N}_{3} \mathrm{CL}-g \text {-PONBIMC }\right)_{10}$ polymer to interact with the biological systems, such as in drug delivery development, tissue engineering, and biomedical material synthesis. These tests are typically conducted by mixing GlucoONB-P $\left(\alpha \mathrm{N}_{3} \mathrm{CL}-g \text {-PONBIMC }\right)_{10}$ with a lectin that is selective for the sugar conjugated to the polymer. ${ }^{35} \mathrm{~A}$ positive result is
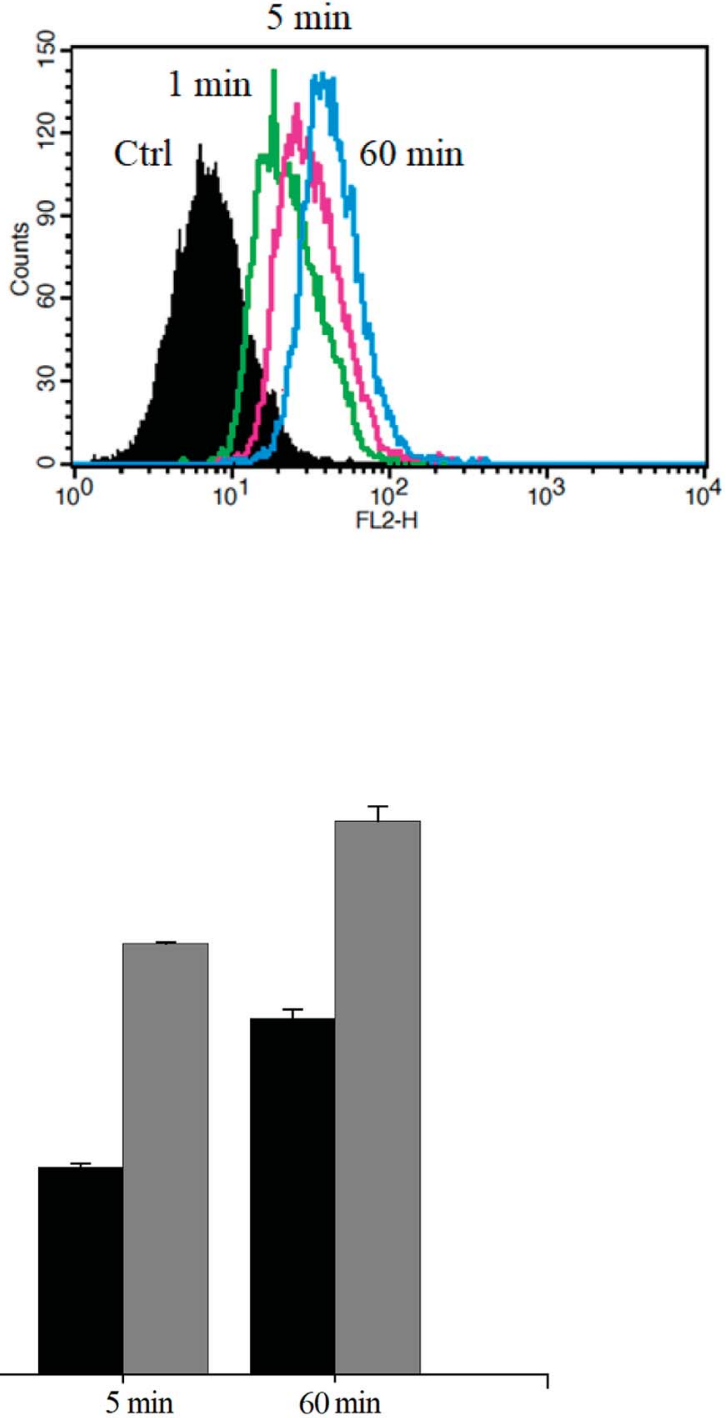

Incubation time obtained when a precipitate appears because of lectin aggregation; this can be measured as the reduction in the transparency of the solution. Con $\mathrm{A}$ is a specific lectin used for selective binding to glucosyl residues. Therefore, we investigated the change in absorbance of solutions of Gluco-ONB$\mathrm{P}\left(\alpha \mathrm{N}_{3} \mathrm{CL}-g \text {-PONBIMC }\right)_{10}$ with Con A at $600 \mathrm{~nm}$. Fig. 10 illustrates that the absorbance (i.e., turbidity) increased with the concentration of Gluco-ONB-P $\left(\alpha \mathrm{N}_{3} \mathrm{CL}-g \text {-PONBIMC }\right)_{10}$ because of the formation of larger aggregates. For a control reading, a PBS buffer solution without Con A was added to the glucose polymer. A marginal absorbance significantly lower than that for the sample containing Con A, was detected. ${ }^{36}$ These experiments confirmed that Gluco-ONB-P $\left(\alpha \mathrm{N}_{3} \mathrm{CL}-g \text {-PONBIMC }\right)_{10}$ synthesized 
through the nucleophilic coupling of Gluco- $\left(\mathrm{CH}_{2}\right)_{3} \mathrm{Br}$ to HONB$\mathrm{P}\left(\alpha \mathrm{N}_{3} \mathrm{CL}-g \text {-PONBIMC }\right)_{10}$ entailed active biorecognition.

\section{In vitro cytotoxicities of the polymer}

Cytotoxicity is a crucial consideration in drug carrier design. In vitro cytotoxicities of the Gluco-ONB-P $\left(\alpha \mathrm{N}_{3} \mathrm{CL}-g \text {-PONBIMC }\right)_{10}$ polymer with and without UV irradiation were evaluated through a 3-(4,5-dimethylthiazol-2-yl)-5-(3-carboxymethoxyphenyl)-2-(4sulfophenyl)-2H-tetrazolium (MTS) assay of HeLa cells treated with various polymer concentrations. To eliminate the undesirable cytotoxic effects of the polymer with and without UV irradiation, the viability of cells loaded with increasing amounts of Gluco-ONB-P( $\alpha \mathrm{N}_{3} \mathrm{CL}-g$-PONBIMC $)_{10}$ was assessed using a Promega CellTiter $96 \circledast$ AQueuous One Solution kit. HeLa cells were incubated with various concentrations of Gluco-ONB-P $\left(\alpha \mathrm{N}_{3} \mathrm{CL}-g\right.$ PONBIMC $)_{10}$ for $48 \mathrm{~h}$ followed by a reaction with the MTS reagent, which was bioreduced to formazan because of the esterase in living cells, enabling spectrophotometric analysis absorbance at $485 \mathrm{~nm}$. Fig. 11A depicts the relative viability percentages of cells treated with various concentrations of GlucoONB-P $\left(\alpha \mathrm{N}_{3} \mathrm{CL}-g \text {-PONBIMC }\right)_{10}$ before UV exposure and after $1 \mathrm{~h}$ of UV irradiation for $48 \mathrm{~h}$. Cell viability was $80 \%$ higher than the control at a polymer concentration ranging from 1 to $30 \mu \mathrm{g} \mathrm{mL} \mathrm{L}^{-1}$. The results demonstrate that the Gluco-ONB-P $\left(\alpha \mathrm{N}_{3} \mathrm{CL}-\mathrm{g}\right.$ PONBIMC) $)_{10}$ solution before UV exposure and after $1 \mathrm{~h}$ of UV irradiation were slightly cytotoxic. In addition, Fig. 11B presents the in vitro cytotoxicities of the DOX-loaded micelles and free DOX at various DOX dosages $\left(0.125-10 \mu \mathrm{g} \mathrm{mL}^{-1}\right)$. The DOXloaded Gluco-ONB-P $\left(\alpha \mathrm{N}_{3} \mathrm{CL}-g \text {-PONBIMC }\right)_{10}$ micelles effectively in-habited HeLa cell proliferation with a half-maximal inhibitory concentration $\left(\mathrm{IC}_{50}\right)$ of $8.8 \mu \mathrm{g} \mathrm{mL} \mathrm{m}^{-1}$. The DOX-loaded micelles possess higher $\mathrm{IC}_{50}$ than the free DOX $\left(2.4 \mu \mathrm{g} \mathrm{mL} \mathrm{m}^{-1}\right)$, which is observed in many polymeric system. ${ }^{37}$ This is likely caused by the longer time required for DOX to be released from micelles into tumor cells.

\section{Cellular uptake profile of doxorubicin-incorporated micelles}

The cellular uptake of prodrug micelles by HeLa cells was investigated through flow cytometry and confocal laser scanning microscopy (CLSM). Amphiphilic drug-conjugated polymer can transport drugs through micelle formation. We investigated the drug-carrying ability of the polymeric GlucoONB-P $\left(\alpha \mathrm{N}_{3} \mathrm{CL}-\mathrm{g} \text {-PONBIMC }\right)_{10}$ micelles using DOX, a potent antitumor drug. The self-fluorescence characteristic of this drug facilitated the identification and quantification of drugincorporated micelles that entered the cells. ${ }^{38,39}$

DOX-incorporated Gluco-ONB-P( $\alpha \mathrm{N}_{3} \mathrm{CL}-g$-PONBIMC $)_{10}$ micelles were prepared through dialysis, and the uptake of micelles and free DOX in equal concentration (458.2 $\mathrm{ng} \mathrm{mL}^{-1}$ ) at 1,5 , and $60 \mathrm{~min}$, respectively, were recorded through flow cytometry. In the first $5 \mathrm{~min}$, DOX uptake in the cells incubated with DOX-incorporated micelles was similar to that in those treated with free DOX (Fig. 12A). However, DOX in its free-form enters and accumulates into cells faster than the DOX encapsulated in micelles at incubation time of up to $60 \mathrm{~min}$. The geometric mean fluorescence intensity in the HeLa cells treated with DOX-incorporated Gluco-ONB-P $\left(\alpha \mathrm{N}_{3} \mathrm{CL} \text {-g-PONBIMC }\right)_{10}$ was approximately 0.6-fold weaker than that in the HeLa cells treated with free DOX during the $60 \mathrm{~min}$ incubation time (Fig. 12B). Various fluorescence microscopic experiments were conducted to determine the intracellular distributions of DOXincorporated Gluco-ONB-P $\left(\alpha \mathrm{N}_{3} \mathrm{CL}-g \text {-PONBIMC }\right)_{10}$ micelles and free DOX following the cellular entry. Fig. 13 depicts the results of the nuclear stained Hoechst 33342 and DOX fluorescence as
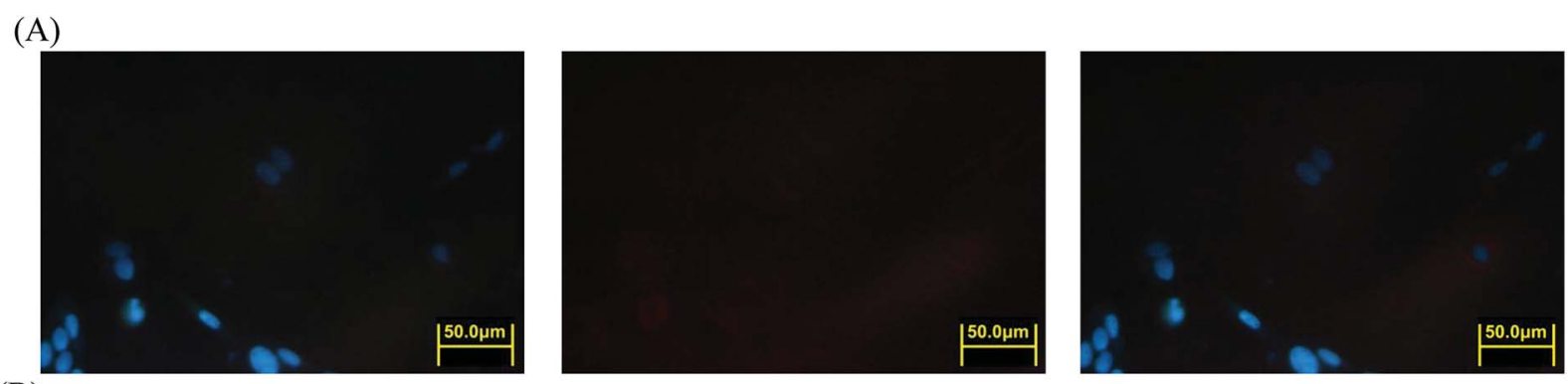

(B)
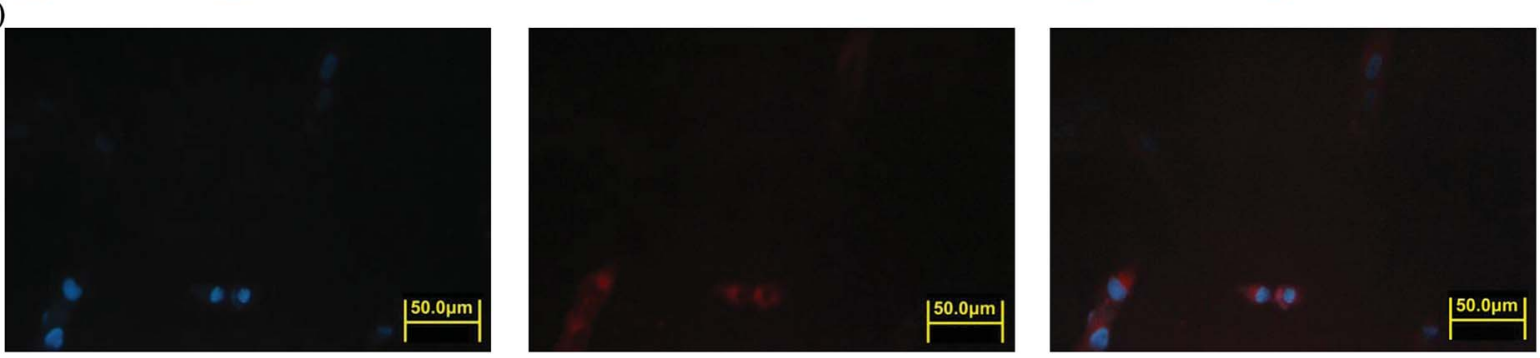

Fig. 15 Fluorescent microscopic images of HeLa cells incubated with free DOX (254.7 ng $\mathrm{mL}^{-1}$ ) or after 5 min UV irradiation DOX-loaded GlucoONB-P $\left(\alpha N_{3} C L-g-P O N B I M C\right)_{10}$ micelles for 60 min: (A) free DOX, and (B) after UV irradiation DOX-loaded micelles. For each row, images for left to right show the cells with Hoechst 33342 nuclear staining, DOX fluorescence, and the merged image (scale bar $50 \mu \mathrm{m}$; brightness not proportional to fluorescence intensity). 
well as an overlay of the images (from left to right). These images indicate that free DOX and Gluco-ONB-P $\left(\alpha \mathrm{N}_{3} \mathrm{CL}-g\right.$ PONBIMC) $)_{10}$-encapsulated DOX exhibited distinct temporal and spatial entry patterns. Free DOX accumulated in the cells at a substantially faster rate than did the Gluco-ONB-P $\left(\alpha \mathrm{N}_{3} \mathrm{CL}-g\right.$ PONBIMC) $)_{10}$-encapsulated DOX, with a minimal fluorescence after $1 \mathrm{~min}$ of treatment, yielding visible fluorescence after $60 \mathrm{~min}$ of treatment (Fig. 13A and C). DOX fluorescence was predominantly concentrated in the cell nuclei, which could be an inherent tendency of the free DOX. ${ }^{40}$ By contrast, in the HeLa cells treated using DOX-incorporated Gluco-ONB-P $\left(\alpha \mathrm{N}_{3} \mathrm{CL}-g\right.$ PONBIMC) $)_{10}$ for 1 or $60 \mathrm{~min}$, DOX fluorescence was concentrated in the cytoplasm with little to no DOX visible in the nucleus (Fig. 13B and D). These differences are attributable to the free DOX entering the cells through passive diffusion, whereas the Gluco-ONB-P $\left(\alpha N_{3} \text { CL- } g \text {-PONBIMC }\right)_{10}$ micelleencapsulated DOX penetrated the plasma membrane through endocytosis. ${ }^{41}$ Although the uptake of the DOX-incorporated Gluco-ONB-P $\left(\alpha \mathrm{N}_{3} \mathrm{CL} \text { - } g \text {-PONBIMC }\right)_{10}$ micelles was slow, the intensity of nuclear fluorescence in the HeLa cells treated with DOX-incorporated Gluco-ONB-P $\left(\alpha \mathrm{N}_{3} \mathrm{CL}-g \text {-PONBIMC }\right)_{10}$ was marginally higher after $60 \mathrm{~min}$ than after $1 \mathrm{~min}$ (Fig. 13B and D). Entry into the nuclei by DOX confirmed that Gluco-ONB$\mathrm{P}\left(\alpha \mathrm{N}_{3} \mathrm{CL} \text { - } g \text {-PONBIMC }\right)_{10}$ micelle-encapsulated DOX was released and successfully reached its pharmacological target. Low penetration of DOX-incorporated Gluco-ONB-P $\left(\alpha \mathrm{N}_{3} \mathrm{CL}-\mathrm{g}\right.$ PONBIMC $)_{10}$ micelles into the extravascular tumor tissues may be attributed primarily to bulky micelle systems being substantial hindered from penetrating into tumor cells. ${ }^{42}$

The intracellular DOX release from DOX-incorporated GlucoONB-P $\left(\alpha \mathrm{N}_{3} \mathrm{CL}-\mathrm{g} \text {-PONBIMC }\right)_{10}$ under UV irradiation was also confirmed by flow cytometry and CLSM studies. As observed from Fig. 14 and 15, HeLa cells incubated with DOXincorporated Gluco-ONB-P $\left(\alpha \mathrm{N}_{3} \mathrm{CL} \text { - } g \text {-PONBIMC }\right)_{10}$ micelles after UV irradiation for 5 min displayed stronger intracellular DOX fluorescence intensity as compared to the free DOX. The DOX-incorporated micelles after UV irradiation for 5 min enters and accumulates into cells faster than the free-form DOX during the incubated time (Fig. 14A). The geometric mean fluorescence intensity in the HeLa cells treated with DOX-incorporated Gluco-ONB-P $\left(\alpha \mathrm{N}_{3} \mathrm{CL} \text { - } g \text {-PONBIMC }\right)_{10}$ under UV irradiation was approximately 1.6-fold stronger than that in the HeLa cells treated with free DOX at incubation time of up to $60 \mathrm{~min}$ (Fig. 14B). Fig. 15 depicts the results of the nuclear stained Hoechst 33342 and DOX fluorescence as well as an overlay of the images (from left to right). These images indicate that the HeLa cells treated using DOX-incorporated micelles after UV irradiation for $60 \mathrm{~min}$, DOX fluorescence was major concentrated in the cytoplasm. These results displayed that the cellular uptake of prodrug micelles under UV irradiation enters and accumulates into cells faster than without UV irradiation. ${ }^{43}$

\section{Conclusions}

A family of phototriggered amphiphilic block-grafted copolymers and conjugated IMC in the hydrophobic segment prodrug were synthesized. The copolymers and prodrug with a photocleavable junction points between the hydrophilic glycose and the hydrophobic $\mathrm{P}\left(\alpha \mathrm{N}_{3} \mathrm{CL} \text { - } g \text {-alkyne }\right)_{n}$ blocks were characterized through ${ }^{1} \mathrm{H}$ NMR, FTIR, and GPC. The obtained copolymers and prodrug formed micelles with a spherical morphology in an aqueous solution. UV irradiation burst release of the encapsulated or conjugated drug. Cell viability was evaluated in response to these particles at concentrations of $\leq 1000 \mu \mathrm{g} \mathrm{mL}{ }^{-1}$, with toxicity to HeLa cells when the concentration was $\geqq 100 \mu \mathrm{g} \mathrm{mL} \mathrm{mL}^{-1}$. After UV irradiation Gluco-ONB$\mathrm{P}\left(\alpha \mathrm{N}_{3} \mathrm{CL} \text { - } g \text {-PONBIMC }\right)_{10}$ encapsulated DOX micelles entered and accumulated in the cells faster than its free-form counterpart and without UV irradiation. Thus, the results suggested that light-sensitive Gluco-ONB-P $\left(\alpha \mathrm{N}_{3} \mathrm{CL}-g \text {-PONBIMC }\right)_{10}$ prodrug has potential targeted drug delivery applications.

\section{Conflicts of interest}

There are no conflicts to declare.

\section{Acknowledgements}

The research was supported by grants from Chang Gung Memorial Hospital (CMRPD5F0012) and the authors thank the Microscopy Center at Chang Gung University for technical assistance.

\section{References}

1 E. Fleige, M. A. Quadir and R. Haag, Adv. Drug Delivery Rev., 2012, 64, 866-884.

2 N. Kamaly, Z. Xiao, P. M. Valencia, A. F. Radovic-Moreno and O. C. Farokhzad, Chem. Soc. Rev., 2012, 41, 2971-3010.

3 A. Rösler, G. W. M. Vandermeulen and H. A. Klok, Adv. Drug Delivery Rev., 2012, 64, 270-279.

4 T. M. Allen and P. R. Cullis, Adv. Drug Delivery Rev., 2013, 65, 36-48.

5 A. Aoleimani, A. Borecki and E. R. Gillies, Polym. Chem., 2014, 5, 7062-7071.

6 V. Delplace, P. Couvreur and J. Nicolas, Polym. Chem., 2014, 5, 1529-1544.

7 Y. Bao, E. Guégain, J. Mougin and J. Nicolas, Polym. Chem., 2018, 9, 687-698.

8 L. Zhou, M. Chen, Y. Guan and Y. Zhang, Polym. Chem., 2014, 5, 7081-7089.

9 Z. Wang, Q. He, W. Zhao, J. Luo and W. Gao, J. Controlled Release, 2017, 264, 66-75.

10 S. Chen, Q. Bian, P. Wang, X. Zheng, L. Lv, Z. Dang and G. Wang, Polym. Chem., 2017, 8, 6150-6157.

11 Sauraj, S. U. Kumar, V. Kumar, R. Priyadarshi, P. Gopinath and Y. S. Negi, Carbohydr. Polym., 2018, 188, 252-259.

12 C. Englert, I. Nischang, C. Bader, P. Borchers, J. Alex, M. Pröhl, M. Hentschel, M. Hartlieb, A. Traeger, G. Pohnert, S. Schubert, M. Gottschaldt and U. S. Schubert, Angew. Chem., Int. Ed., 2018, 57, 2479-2482.

13 R. Zhang, X. Jia, M. Pei and P. Liu, React. Funct. Polym., 2017, 116, 24-30. 
14 T. Zhou, X. Jia, X. Zhao, J. Li and P. Liu, J. Mater. Chem. B, 2017, 5, 2840-2848.

15 Y. Yu, C. K. Chen, W. C. Law, H. Sun, P. N. Prasad and C. Cheng, Polym. Chem., 2015, 6, 953-961.

16 O. Bertrand and J. F. Gohy, Polym. Chem., 2017, 8, 52-73.

17 Q. Jin, F. Mitschang and S. Agarwal, Biomacromolecules, 2011, 12, 3684-3691.

18 Y. Shamay, L. Adar, G. Ashkenasy and A. David, Biomaterials, 2011, 32, 1377-1386.

19 X. Tan, B. B. Li, X. Lu, F. Jia, C. Santori, P. Menon, H. Li, B. Zhang, J. J. Zhao and K. Zhang, J. Am. Chem. Soc., 2015, 137, 6112-6115.

20 S. Lal, S. E. Clare and N. J. Halas, Acc. Chem. Res., 2008, 41, 1842-1851.

21 D. A. Ossipov, A. B. Romero and E. Ossipova, Carbohydr. Polym., 2018, 180, 145-155.

22 S. Lenior, R. Riva, X. Lou, Ch. Detrembleur, R. Jérôme and P. Lecomte, Macromolecules, 2004, 37, 4055-4061.

23 R. S. Lee, Y. C. Li and S. W. Wang, Carbohydr. Polym., 2015, 117, 201-210.

24 C. Giacomelli, V. Schmidt and R. Borsali, Macromolecules, 2007, 40, 2148-2157.

25 C. Lu, Q. Shi, X. Chen, T. Lu, Z. Xie, X. Hu, J. Ma and X. Jing, J. Polym. Sci., Part A: Polym. Chem., 2007, 45, 3204-3217.

26 H. Kuang, S. Wu, Z. Xie, F. Meng, X. Jing and Y. Huang, Biomacromolecules, 2012, 13, 3004-3012.

27 X. Hu, J. Tian, T. Liu, G. Zhang and S. Liu, Macromolecules, 2013, 46, 6243-6256.

28 D. R. Griffin, J. L. Schlosser, S. F. Lam, T. H. Nguyen, H. D. Maynard and A. M. Kasko, Biomacromolecules, 2013, 14, 1199-1207.
29 Y. Dong and S. S. Feng, Biomaterials, 2004, 25, 2843-2849.

30 A. Kataoka, A. Harada and Y. Nagasaki, Adv. Drug Delivery Rev., 2001, 47, 113-131.

31 Y. Zhang, J. He, D. Cao, M. Zhang and P. Ni, Polym. Chem., 2014, 5, 5124-5138.

32 C. Yang, A. B. Ebrahim attia, J. P. K. Tan, X. Ke, S. Gao and J. L. Hedrick, Biomaterials, 2012, 33, 2971-2979.

33 C. Lv, Z. Wang, P. Wang and X. Tang, Langmuir, 2012, 28, 9387-9394.

34 P. M. Antonik, A. M. Eissa, A. R. Round and N. R. Cameron, Biomacromolecules, 2016, 17, 2719-2725.

35 M. Ambrosi, N. R. Cameron and B. G. Davis, Org. Biomol. Chem., 2005, 3, 1593-1608.

36 J. Huang, G. Habraken, F. Audouin and A. Heise, Macromolecules, 2010, 43, 6050-6057.

37 Y. Wu, D. Zhou, Y. Qi, Z. Xie, X. Chen, X. Jing and Y. Huang, RSC Adv., 2015, 5, 31972-31983.

38 X. Dai, Z. Yue, M. E. Eccleston, J. Swartling, N. K. Slater and C. F. Kaminski, Nanomedicine, 2008, 4, 49-56.

39 Y. Jin, L. Song, Y. Su, L. Zhu, Y. Peng and F. Qiu, Biomacromolecules, 2011, 12, 3460-3468.

40 M. Sui, W. Liu and Y. Shen, J. Controlled Release, 2011, 155, 227-236.

41 J. Yan, Z. Ye, M. Chen, Z. Liu, Y. Xiao and Y. Zhang, Biomacromolecules, 2011, 12, 2562-2572.

42 S. M. Sagnella, H. Duong, A. MacMillan, C. Boyer, R. Whan and J. A. McCarroll, Biomacromolecules, 2013, 15, 262-275.

43 Y. Zhang, C. Y. Ang, M. Li, S. Y. Tan, Q. Qu, Z. Luo and Y. Zhao, ACS Appl. Mater. Interfaces, 2015, 7, 18179-18187. 